\title{
Spectral Densities of Response Functions for the O(3) Symmetric Anderson and Two Channel Kondo Models
}

\author{
S.C. Bradley ${ }^{1}$, R. Bulla ${ }^{2}$, A.C. Hewson ${ }^{1}$, and G-M. Zhang ${ }^{3}$ \\ ${ }^{1}$ Department of Mathematics, Imperial College, London SW7 2BZ, UK. \\ ${ }^{2}$ Theoretische Physik III, Elektronische Korrelationen und Magnetismus, Universtität Augsburg, 86135 Augsburg, \\ Germany \\ ${ }^{3}$ Centre for Advanced Study, Tsinghua University, Beijing 100084, P.R. of China
}

\begin{abstract}
The $\mathrm{O}(3)$ symmetric Anderson model is an example of a system which has a stable low energy marginal Fermi liquid fixed point for a certain choice of parameters. It is also exactly equivalent, in the large $U$ limit, to a localized model which describes the spin degrees of freedom of the linear dispersion two channel Kondo model. We first use an argument based on conformal field theory to establish this precise equivalence with the two channel model. We then use the numerical renormalization group (NRG) approach to calculate both one-electron and two-electron response functions for a range of values of the interaction strength $U$. We compare the behaviours about the marginal Fermi liquid and Fermi liquid fixed points and interpret the results in terms of a renormalized Majorana fermion picture of the elementary excitations. In the marginal Fermi liquid case the spectral densities of all the Majorana fermion modes display a $|\omega|$ dependence on the lowest energy scale, and in addition the zero Majorana mode has a delta function contribution. The weight of this delta function is studied as a function of the interaction $U$ and is found to decrease exponentially with $U$ for large $U$. Using the equivalence with the two channel Kondo model in the large $U$ limit, we deduce the dynamical spin susceptibility of the two channel Kondo model over the full frequency range. We use renormalized perturbation theory to interpret the results and to calculate the coefficient of the $\ln \omega$ divergence found in the low frequency behaviour of the $T=0$ dynamic susceptibility.
\end{abstract}

\section{INTRODUCTION}

The $\mathrm{O}(3)$ symmetric Anderson model is a modified form of the symmetric Anderson model which was introduced by Coleman and Schofieldl. The Hamiltonian can be expressed in the form,

$$
\begin{gathered}
H=\sum_{n=0, \sigma}^{\infty} t_{n}\left(c_{n+1, \sigma}^{\dagger} c_{n \sigma}+c_{n \sigma}^{\dagger} c_{n+1, \sigma}\right) \\
+\sum_{\sigma} V_{\sigma}\left(c_{0 \sigma}^{\dagger} c_{d \sigma}+c_{d \sigma}^{\dagger} c_{0 \sigma}\right)+V_{2}\left(c_{d \downarrow}^{\dagger} c_{0 \downarrow}^{\dagger}+c_{0 \downarrow} c_{d \downarrow}\right) \\
+U\left(n_{d \uparrow}-\frac{1}{2}\right)\left(n_{d \downarrow}-\frac{1}{2}\right),
\end{gathered}
$$

where $c_{d \sigma}^{\dagger}$ and $c_{d \sigma}$ are creation and annihilation operators for the localized impurity d state with spin $\sigma$, with $V_{\uparrow}=V$, $V_{\downarrow}=\left(V_{0}+V\right) / 2$ and $V_{2}=\left(V_{0}-V\right) / 2$. The electrons in the impurity state interact via a Coulomb matrix element $U$, and are coupled by the hybridization terms to the conduction electrons which are in the form of a tight-binding chain with $c_{n \sigma}^{\dagger}, c_{n \sigma}$, the creation and annihilation operators at site $n$, and $t_{n}$ is the nearest neighbour hopping matrix element. The model is basically a symmetric Anderson model $\left(V=V_{0}\right)$ with an anomalous hybridization term $\left(V \neq V_{0}\right)$ which breaks both spin and charge conservation. It takes a simpler form when expressed in terms of Majorana fermions operators. For the impurity d electrons the Majorana fermion operators are defined by

$$
\begin{aligned}
& d_{1}=\frac{1}{\sqrt{2}}\left(c_{d \uparrow}+c_{d \uparrow}^{\dagger}\right) \quad d_{2}=\frac{i}{\sqrt{2}}\left(c_{d \uparrow}-c_{d \uparrow}^{\dagger}\right), \\
& d_{3}=\frac{-1}{\sqrt{2}}\left(c_{d \downarrow}+c_{d \downarrow}^{\dagger}\right) \quad d_{0}=\frac{i}{\sqrt{2}}\left(c_{d \downarrow}-c_{d \downarrow}^{\dagger}\right),
\end{aligned}
$$

which satisfy the commutation relations,

$$
\left\{d_{\alpha}, d_{\beta}\right\}=\delta_{\alpha, \beta}
$$


where \{\} indicates an anticommutator.

The Majorana operators for the conduction electrons are similarly defined by

$$
\begin{aligned}
\chi_{1}(n) & =\frac{e^{i n \pi / 2}}{\sqrt{2}}\left((-1)^{n} c_{n \uparrow}+c_{n \uparrow}^{\dagger}\right), \\
\chi_{2}(n) & =\frac{i e^{i n \pi / 2}}{\sqrt{2}}\left((-1)^{n} c_{n \uparrow}-c_{n \uparrow}^{\dagger}\right), \\
\chi_{3}(n) & =\frac{-e^{i n \pi / 2}}{\sqrt{2}}\left((-1)^{n} c_{n \downarrow}+c_{n \downarrow}^{\dagger}\right) \\
\chi_{0}(n) & =\frac{i e^{i n \pi / 2}}{\sqrt{2}}\left((-1)^{n} c_{n \downarrow}-c_{n \downarrow}^{\dagger}\right),
\end{aligned}
$$

with commutation relations as in (5).

Using the Majorana fermion representation the $\mathrm{O}(3)$ Anderson model can be expressed in the form

$$
\begin{gathered}
H=i \sum_{\alpha=0}^{3} V \chi_{\alpha}(0) d_{\alpha}+i V_{0} \chi_{0}(0) d_{0} \\
+i \sum_{\alpha=0}^{3} \sum_{n=0}^{\infty} t_{n} \chi_{\alpha}(n+1) \chi_{\alpha}(n)+U d_{1} d_{2} d_{3} d_{0},
\end{gathered}
$$

which is clearly invariant under the $\mathrm{O}(3)$ group of transformations of the basis of the 1,2,3 Majorana fermions.

There are two aspects of this model that make it worthy of study. One is that the model displays non-Fermi liquid behaviour corresponding to marginal Fermi liquid theory in lowest order perturbation theory for $V_{0}=0^{2}$. The marginal behaviour results from singular scattering with an uncoupled local Majorana fermion mode. Numerical renormalization group (NRG) calculations 3 have established that this marginal Fermi liquid behaviour persists down to $T=0$ and that there is an associated residual entropy of $\ln 2 / 2$. This fixed point behaviour has been interpreted in terms of the free renormalized Majorana fermion modes. The many-body excitations constructed from these free Majorana fermions account for the excitations found at the fixed point in the NRG calculations $\mathbb{A}$. For $V_{0} \neq 0$ there is no uncoupled local Majorana fermion mode and the thermodynamic behaviour corresponds to Fermi liquid theory in terms of renormalized Majorana fermions.

The original reason Coleman and Schofield 1 studied this model is because, in the large $U$ regime under a SchriefferWolff transformation, it maps into a localized spin model, the $\sigma-\tau$ model 5 , which has a similar form to the two channel Kondo model (TCKM). The difference is that the localized spin $S=1 / 2$ is coupled via an exchange interaction to the spin and the isospin of conduction electrons in a single channel rather than the spins of the conduction electrons in two distinct channels as in the TCKM (for a comprehensive review of this model seed). The Hamiltonian of the model can be written in the form,

$$
H=\vec{S}_{d} \cdot\left[J_{1} \vec{\sigma}(0)+J_{2} \vec{\tau}(0)\right]+\sum_{n=0, \sigma}^{\infty} t_{n}\left(c_{n+1, \sigma}^{\dagger} c_{n \sigma}+c_{n, \sigma}^{\dagger} c_{n+1, \sigma}\right),
$$

where $\vec{S}_{d}$ is the operator for the localized spin and $J_{1}, J_{2}$ are the two exchange couplings.

The spin operators,

$$
\begin{array}{r}
\sigma^{+}(n)=c_{n \uparrow}^{\dagger} c_{n \downarrow}, \quad \sigma^{-}(n)=c_{n \downarrow}^{\dagger} c_{n \uparrow}, \\
\sigma_{\mathrm{z}}(n)=\frac{1}{2}\left(c_{n \uparrow}^{\dagger} c_{n \uparrow}-c_{n \downarrow}^{\dagger} c_{n \downarrow}\right),
\end{array}
$$

in the basis spanned by the two singly occupied fermion states, $|0(\uparrow), 1(\downarrow)\rangle$ and $|1(\uparrow), 0(\downarrow)\rangle$, give a representation for the $\mathrm{SU}(2)$ algebra for spin $\sigma=\frac{1}{2}$. The isospin operators,

$$
\begin{gathered}
\tau^{+}(n)=(-1)^{n} c_{n \uparrow}^{\dagger} c_{n \downarrow}^{\dagger}, \quad \tau^{-}(n)=(-1)^{n} c_{n \downarrow} c_{n \uparrow}, \\
\tau_{\mathrm{z}}(n)=\frac{1}{2}\left(c_{n \uparrow}^{\dagger} c_{n \uparrow}+c_{n \downarrow}^{\dagger} c_{n \downarrow}-1\right),
\end{gathered}
$$


give a representation of the same algebra in the space spanned by the zero and double occupation states, $|0,0\rangle$ and $|1,1\rangle$. By applying the Schrieffer-Wolff transformation it can be shown that the $\sigma$ - $\tau$ model corresponds to the localized or large $U$ limit of the $\mathrm{O}(3)$ Anderson model with the coupling $J_{1}$ and $J_{2}$ given by

$$
J_{1}=\frac{2 V\left(V+V_{0}\right)}{U} \quad J_{2}=\frac{2 V\left(V-V_{0}\right)}{U} .
$$

The channel isotropic model $J_{1}=J_{2}=J$, corresponds to $V_{0}=0$ in the $\mathrm{O}(3)$ Anderson model. In this case one of the Majorana fermions modes is uncoupled and the singular scattering of the conduction electrons gives rise to a marginal Fermi liquid fixed point. In terms of Majorana fermions the $\sigma-\tau$ model (12) takes the form,

$$
H=i \sum_{\alpha=0}^{3} \sum_{n=0}^{\infty} t_{n} \chi_{\alpha}(n+1) \chi_{\alpha}(n)-\frac{i J}{2} \vec{S}_{d} \cdot \vec{\chi}(0) \times \vec{\chi}(0),
$$

where $\vec{\chi}(n)=\left(\chi_{-1}(n), \chi_{2}(n), \chi_{3}(n)\right)$.

Coleman et al 5 put forward arguments that this one band localized model has the same fixed point and low energy behaviour as the two channel Kondo model. A bosonization approash by Schofield supported this conjecture, and so did a comparision of our numerical renormalization group results 3 for the large $U \mathrm{O}(3)$ Anderson model with the results for the two channel model.

In the first section of this paper we show that if we assume linear dispersion for the conduction electrons the relationship between the $\sigma-\tau$ model and the spin degrees of freedom of the two channel model becomes an exact one, and what is more it is not confined purely to the asymptotic low energy regime but applies to all relevant energy scales. In the subsequent sections we extend our earlier numerical renormalization group calculations 3 for the $\mathrm{O}(3)$ Anderson model to the calculation the dynamical response functions at $T=0$ both for the marginal Fermi liquid and Fermi liquid cases, and interpret these results using renormalized perturbation theory for the Majorana fermions䁬. Finally we use the equivalence of the $\mathrm{O}(3)$ Anderson model and the two channel Kondo model in the large $U$ limit to deduce the form of the dynamic spin susceptibility for the two channel Kondo model over all relevant energy scales. We use renormalized perturbation theory to deduce the coefficient of the $\ln \omega$ divergence found in the low frequency behaviour of the real part of the dynamic susceptibility at zero temperature.

\section{EXACT EQUIVALENCE TO THE TWO CHANNEL KONDO MODEL}

We begin by showing that the Majorana fermion description, used to formulate the $\mathrm{O}(3)$ Anderson model and $\sigma-\tau$ models, can emerge quite naturally for the two channel model as a representation of the algebra of the total spin current of the two channels. For linear dispersion and no cut-off we can express the Hamiltonian of the channel isotropic TCKM model in the form,

$$
\begin{aligned}
& H=H_{0}+H_{I} \\
& H_{0}=\frac{v_{f}}{2 \pi} \sum_{j=1}^{2} \sum_{\sigma=\uparrow, \downarrow} \int_{-\infty}^{+\infty} d x: \psi_{j, \sigma}^{\dagger}(x)\left(i \partial_{x}\right) \psi_{j, \sigma}(x): \\
& H_{I}=\sum_{a=x, y, z} J_{a} S_{d}^{a} J_{s}^{a}(0),
\end{aligned}
$$

on taking a Fourier transform to a continuum variable $x$, where $v_{f}$ is the Fermi velocity, $j$ is a channel index, and the conduction electron operators between the colons have to be to normal ordered. We have assumed s-wave scattering only and replaced the incoming and outgoing waves with two left-moving electron fields $\psi_{j, \sigma}(x) ; J_{s}^{a}(x)$ are the conduction electron spin current operators

$$
J_{s}^{a}(x)=\sum_{j, \sigma, \sigma^{\prime}}: \psi_{j, \sigma}^{\dagger}(x) s_{\sigma, \sigma^{\prime}}^{a} \psi_{j, \sigma^{\prime}}(x):
$$

$s^{a}$ being spin-1/2 matrices. We can also introduce charge and flavour currents

$$
\begin{aligned}
& J_{c}(x)=\sum_{j, \sigma}: \psi_{j, \sigma}^{\dagger}(x) \psi_{j, \sigma}(x): \\
& J_{f}^{a}(x)=\sum_{j, j^{\prime}, \sigma}: \psi_{j, \sigma}^{\dagger}(x) t_{j, j^{\prime}}^{a} \psi_{j^{\prime}, \sigma}(x): .
\end{aligned}
$$


where $t_{j, j^{\prime}}^{a}$ are generators of an SU(2) symmetry group. Following Affleck and Ludwig 10 , the free part of the Hamiltonian can be rewritten as a sum of three commuting terms by the usual point-splitting procedure (Sugawara construction):

$$
\begin{aligned}
H_{0}=\frac{v_{f}}{2 \pi} \int_{-\infty}^{+\infty} d x & {\left[\frac{1}{8}: J_{c}(x) J_{c}(x):+\frac{1}{4}: \vec{J}_{f}(x) \cdot \vec{J}_{f}(x):\right.} \\
+ & \left.\frac{1}{4}: \vec{J}_{s}(x) \cdot \vec{J}_{s}(x):\right],
\end{aligned}
$$

while the interaction term is expressed in terms of the electron spin currents and the impurity spin only. The information about the number of channels is contained in the commutation relations obeyed by the spin currents

$$
\left[J_{s}^{a}(x), J_{s}^{b}\left(x^{\prime}\right)\right]=i \epsilon^{a b c} J_{s}^{a}(x) \delta\left(x-x^{\prime}\right)+\frac{k i}{4 \pi} \delta_{a, b} \delta^{\prime}\left(x-x^{\prime}\right)
$$

indicating that $J_{s}^{a}(x)$ form an $\mathrm{SU}(2)$ level $k=2$ Kac-Moody algebra. Meanwhile, the charge and flavour currents satisfy

$$
\begin{aligned}
& {\left[J_{c}(x), J_{c}\left(x^{\prime}\right)\right]=2 k i \delta^{\prime}\left(x-x^{\prime}\right),} \\
& {\left[J_{f}^{a}(x), J_{f}^{b}\left(x^{\prime}\right)\right]=i \epsilon^{a b c} J_{f}^{a}(x) \delta\left(x-x^{\prime}\right)} \\
& +\frac{k i}{4 \pi} \delta_{a, b} \delta^{\prime}\left(x-x^{\prime}\right) .
\end{aligned}
$$

They form a U(1) Kac-Moody and another SU(2) level-2 Kac-Moody algebra, separately.

It is now quite natural to introduce a Majorana representation of the spin current operators in the form,

$$
\begin{aligned}
& J_{s}^{x}(x)=-i: \chi_{2}(x) \chi_{3}(x): \\
& J_{s}^{y}(x)=-i: \chi_{3}(x) \chi_{1}(x): \\
& J_{s}^{z}(x)=-i: \chi_{1}(x) \chi_{2}(x):
\end{aligned}
$$

where $\chi_{1}(x), \chi_{2}(x)$, and $\chi_{3}(x)$ are left-moving free Majorana fermion fields. It can be shown that this representation reproduces the SU(2) level-2 Kaf-Hoody commutation relations. Our approach differs from earlier conformal field theory approaches to the TCKM 14 16, where Majorana fermions are introduced only at a later stage after bosonization. It is important to note that this Majorana representation of the spin currents is only appropriate for the two channel model as it leads to a level-2 algebra. It would be inappropriate for the single channel Kondo model where the corresponding spin current generates a level-1 algebra.

In a similar way, we can also introduce Majorana representations for the flavour currents

$$
\begin{aligned}
& J_{f}^{x}(x)=-i: \chi_{2}^{\prime}(x) \chi_{3}^{\prime}(x):, \\
& J_{f}^{y}(x)=-i: \chi_{3}^{\prime}(x) \chi_{1}^{\prime}(x):, \\
& J_{f}^{z}(x)=-i: \chi_{1}^{\prime}(x) \chi_{2}^{\prime}(x):,
\end{aligned}
$$

which reproduces the commutation relations satisfied by the flavour currents, and

$$
J_{c}(x)=-2 i: \chi_{4}^{\prime}(x) \chi_{5}^{\prime}(x):
$$

can represent the charge current operator. Note that $\chi_{\alpha}^{\prime}$ with $\alpha=1,2,3,4,5$ are also left-moving free Majorana fermion fields. It is well-known that the dynamics of charge, flavour, and spin are completely determined by the commutation relations of the current operators. Though the spin currents of the two channel Kondo model can be represented in terms of three Majorana fermion fields $\chi_{\alpha}(x)(\alpha=1,2,3)$, we emphasize that they can not be given any simple physical interpretation in terms of the original conduction electrons $\psi_{j, \sigma}(x)$.

At this point we have the current operator terms in the Hamiltonian as quartic in the Majorana fields. The Sugawara construction enables one to write kinetic energy terms, which are quadratic in field operators, as quartic terms. This is what was done earlier in writing the free part of the Hamiltonian in form of equation (16), and it is convenient if one is pursuing a purely algebraic approach as used in the conformal field theory 10 . However for our purposes it is more convenient now to perform an inverse Sugawara construction by the usual point-splitting procedure again, and rewrite the terms quartic in the Majorana fermions as kinetic energy terms which are quadratic 11.12 : 


$$
\begin{aligned}
& : J_{c}(x) J_{c}(x):=4 \sum_{\alpha=4}^{5}: \chi_{\alpha}^{\prime}\left(i \partial_{x}\right) \chi_{\alpha}^{\prime}(x) ; \\
& : \vec{J}_{f}(x) \cdot \vec{J}_{f}(x):=2 \sum_{\alpha=1}^{3}: \chi_{\alpha}^{\prime}\left(i \partial_{x}\right) \chi_{\alpha}^{\prime}(x) ; \\
& : \vec{J}_{s}(x) \cdot \vec{J}_{s}(x):=2 \sum_{\alpha=1}^{3}: \chi_{\alpha}\left(i \partial_{x}\right) \chi_{\alpha}(x) .
\end{aligned}
$$

The model Hamiltonian is transformed and divided into the following two parts,

$$
\begin{gathered}
H_{c}+H_{f}=\frac{v_{f}}{4 \pi} \sum_{\alpha=1}^{5} \int_{-\infty}^{+\infty} d x: \chi_{\alpha}^{\prime}(x)\left(i \partial_{x}\right) \chi_{\alpha}^{\prime}(x): \\
H_{s}=\frac{v_{f}}{4 \pi} \sum_{\alpha=1}^{3} \int_{-\infty}^{+\infty} d x: \chi_{\alpha}(x)\left(i \partial_{x}\right) \chi_{\alpha}(x): \\
-\frac{i J}{2} \vec{S}_{d} \cdot: \vec{\chi}(0) \times \vec{\chi}(0):
\end{gathered}
$$

$H_{c}+H_{f}$ describes the non-interacting charge and flavour degrees of freedom. It has a symmetry group $U(1) \otimes S U(2)_{2}=$ $S O(5)$ and is expressed by five free Majorana fermion fields $\chi_{\alpha}^{\prime}(x)(\alpha=1,2,3,4,5) . H_{s}$ is the main part of the model and describes the spin degrees of freedom with three left-moving Majorana fermion fields $\chi_{\alpha}(\alpha=1,2,3)$ interacting with the impurity spin. It has the symmetry $S U(2)_{2}$ or $S O(3)$ so that the full Hamiltonian has the symmetry group $S O(5) \otimes S O(3)$, which is represented by eight different Majorana fermion fields.

In the two channel model Hamiltonian, $H_{s}$ given in equation (24) is the only part which includes an interaction with the impurity spin. This part of the Hamiltonian is exactly equivalent to the vector part of the $\sigma-\tau$ model defined in equation (12). The zero Majorana fermion contribution to (12) is completely decoupled from the other modes so we can separate it out, and if we take the continuum limit with linear dispersion equation (12) can be written in the form,

$$
\begin{aligned}
& H=H_{\mathrm{sc}}+H_{\mathrm{vec}} \\
& H_{\mathrm{sc}}=\frac{v_{f}}{2 \pi} \int_{-\infty}^{+\infty} d x: \chi_{0}(x)\left(i \partial_{x}\right) \chi_{0}(x): \\
& H_{\mathrm{vec}}=\frac{v_{f}}{2 \pi} \sum_{\alpha=1}^{3} \int_{-\infty}^{+\infty} d x: \chi_{\alpha}(x)\left(i \partial_{x}\right) \chi_{\alpha}(x): \\
& \quad-\frac{i J}{2} \vec{S}_{d} \cdot \vec{\chi}(0) \times \vec{\chi}(0): .
\end{aligned}
$$

and can identify $H_{\text {vec }}$ with $H_{s}$. This implies that the application of the $\sigma-\tau$ model is not restricted to the very low energy regime but can be used to calculate the impurity contribution to the thermodynamics of the two channel Kondo model over the full temperature range. This result is exact subject only to the requirement of linear dispersion for the conduction electrons (this is not evident in the approaches that use bosonization 14 16). The result implies that the $\mathrm{O}(3)$ Anderson model in the large $U$ regime can be used to calculate the spin correlation functions of the two channel Kondo model over the complete temperature and frequency range, $\omega, T<<D$, where $D$ is the cut-off imposed on the conduction electrons. We exploit this equivalence to calculate the dynamic susceptibility of the TCKM in the last section of the paper. Though we have restricted our derivation to the channel isotropic case $J_{1}=J_{2}=J$, the result can be generalized to the channel anisotropic case $J_{1} \neq J_{2}$.

The fact that we have been able to establish the exact equivalence the linear dispersion TCKM and $\sigma$ - $\tau$ models, not just for the low energy regime but over the full parameter range of the model, is at first sight a little surprising. A feature of the general TCKM is that there is a strong coupling regime in which the impurity can be overscreened, and this strong coupling fixed point was shown by Noziéres and Blandin 13 to be an unstable one. Impurity overscreening on the other hand cannot occur in the strong coupling regime of the $\sigma-\tau$ model as the spin and isospin are mutually exclusive channels and they cannot both screen the impurity simultaneously. The strong coupling limit for the $\sigma-\tau$ model, in apparent contrast to that of the two channel model, gives a stable fixed point. However, to access the overscreened states of the standard TCKM requires the coupling $J$ to be much greater than the bandwidth $D$. In the linear dispersion two channel model $D \rightarrow \infty$ so these states are not accessible in this version of the two channel 
model. We must distinguish two stong coupling limits. One in which the limit $J \rightarrow \infty$ is taken with a finite cut-off or before the limit $D \rightarrow \infty$ is taken, and one in which the limits are taken in the opposite order. The first leads to overscreening and an unstable fixed point whereas the latter leads to a screened stable fixed point.

A parallel situation arises in the standard Anderson model. If one assumes linear dispersion then the parameter regime in which the impurity level $\epsilon_{d}$ lies outside the conduction band cannot be accessed. The model with the level within the conduction band, and the one with the level below the conduction band, in the Anderson model case have the same fixed point but they do have different expressions for the Kondo temperature (see 9 ).

Before leaving this topic we briefly show that, because the Majorana representation of the TCKM is equivalent to the large $U$ limit of the $\mathrm{O}(3)$ Anderson model, we can use results for the $\mathrm{O}(3)$ model to confirm the description of the TCKM fixed point as one corresponding to renormalized asymptotically free Majorana fermions $5,14,0,15,26$. Our earlier numerical renormalization group calculations 3 established that the fixed point of the $\mathrm{O}(3)$ Anderson model corresponds to free Majorana fermions independent of $U$. This implies that the fixed point corresponds to the noninteracting model $U=0$. The many-body excitations and their degeneracies at the fixed point, as found in the NRG calculations, can be explained in terms of the many body excitations built up from the three non-interacting hybridized 1,2,3 Majorana fermion modes, combined with the excitations arising from the unhybridized zero Majorana fermion modes described by $H_{\mathrm{sc}}$. This interpretation of the fixed point in terms of the $\mathrm{O}(3)$ Anderson model is simpler than the equivalent interpretation as the strong coupling limit of the $\sigma-\tau$ model. In the latter approach a change of boundary condition for the 1,2,3 Majorana fermion modes, which interact with the impurity, has to be invoked as a result of the strong coupling, relative to that of the uncoupled 0 Majorana fermion mode. This is not necessary in the Anderson model description as there is naturally a difference of boundary condition at impurity in the hybridized and unhybridized terms. The leading (dangerously) irrelevent interaction term, which leads to the logarithmically divergent contributions to the specific heat coefficient and susceptibility in these models, can be interpreted as the renormalized interaction term $\bar{U}$ in the Anderson model (seet 5 for further details).

To derive a complete description of the many-body excitations and their degeneracies at the fixed point for the TCKM from the $U=0 \mathrm{O}(3)$ Anderson model we simply have to replace the uncoupled 0 Majorana degree of freedom described by $H_{\mathrm{sc}}$ by the five uncoupled Majorana degrees of freedom in $H_{\mathrm{c}}$ and $H_{\mathrm{f}}$, which describe the charge and flavour excitations. There are two slightly different sets of excitations, one set corresponding to a chain of even sites (sector A) and another set corresponding to an odd number of sites (sector B). When these are combined, as shown in Table 1, they reproduce the many-body energy levels and degeneracies found in the NRG calculations 17 and conformal field theory calculations 10 for the TCKM. Since the interaction is restricted to the spin part of the Hamiltonian $H_{\mathrm{s}}$ the leading (dangerously) irrelevent interaction which leads to logarithmically divergent contributions to the specific heat coefficient and susceptibility in the TCKM is exactly the same as the renormalized interaction $\bar{U} \sim T_{\mathrm{K}}$ of the $\mathrm{O}(3)$ Anderson model, where $T_{\mathrm{K}}$ is the Kondo temperature. We will use this explicitly later in section 4 to calculate the dynamic susceptibility of the TCKM from that of the $\mathrm{O}(3)$ Anderson model.

\section{NUMERICAL RENORMALIZATION GROUP CALCULATIONS}

In this section we extend the numerical renormalization group method to calculate the dynamic features of the $\mathrm{O}(3)$ Anderson model. For the basic details of the NRG method as applied to this model we refer to the earlier paperi where the thermodynamic properties were calculated. Calculation of the dynamic behaviour via the NRG approach is rather more involved because additional matrix elements have also to be calculated in the sequence of iterative diagenalizations. The general details of the NRG approach to the calculation of spectra for impurity models are given in 20.21. Because both charge and spin are not conserved for this model the calculations here differ from the standard case in a number of respects and the relevant details are given in the appendix.

\section{A. Spectral densities of single particle Green's functions}

The spectral density $A_{\sigma}(\omega)$ of the single particle Green's function of spin $\sigma$ at the impurity site is given by

$$
\begin{array}{r}
A_{\sigma}(\omega)=\frac{1}{Z} \sum_{n m}\left|\left\langle n\left|c_{d \sigma}^{\dagger}\right| m\right\rangle\right|^{2} \delta\left(\omega-\left(E_{n}-E_{m}\right)\right) \\
\times\left(e^{-\beta E_{m}}+e^{-\beta E_{n}}\right)
\end{array}
$$


where $E_{n}$ are the exact eigenvalues of the Hamiltonian and $Z$ is the partition function $\left(Z=\sum_{n} \exp \left(-\beta E_{n}\right)\right)$. This is evaluated using the many-body states calculated in the sequence of iterative diagonalizations of the NRG. Because of the decreasing energy scales in the sequence of steps in the NRG, the spectral density for a particular frequency have to be calculated from the step in the sequence corresponding to the appropriate energy scale. The spectrum is then built up from the complete set of NRG steps. Though the method has been developed for finite temperature situations, the procedure is more straightforward and more accurate for the case $T \rightarrow 0$, and we have restricted the calculations here to the low temperature limit. Though a large number of states are retained at each step in the NRG calculation (of the order of 300-500 not including degeneracies), the spectrum is discrete and to obtain a continuous curve a broadening is introduced which decreases logarithmically with the energy scale. The advantages of this approach over alternative methods, such a Monte Carlo, are that it can be used at very low temperatures, can be used for any value of $U$, and the results can be checked against known sum rules and identities. The details of the low energy spectrum gan be calculated to a very high resolution. For further details of the approach we refer to the papers cited earliere2021.

Due to the anomalous hybridization term, the $\mathrm{O}(3)$ Anderson model does not conserve either total spin or total charge. However, the combination of total spin plus total isospin $\mathbf{T}$ is conserved and the many-body states and the eigenstates $|m\rangle$ can be classified using the quantumnumbers $T$ and $T_{z}$ associated with the total of this angular momentum and its z-component fnote that in referencet the symbol $\mathbf{j}$ was used for this quantity, but here we revert to the earlier notation $\mathbf{T}$ used in 322 ). In evaluating (25) the sum over the degeneracies associated with $T_{z}$ can be done explicitly as explained in the appendix. The single particle Green's functions for the impurity can be expressed as linear combinations of the Green's functions for the Majorana fermions, $G_{\alpha \beta}\left(t-t^{\prime}\right)=-i \theta\left(t-t^{\prime}\right)\left\langle\left\{d_{\alpha}(t) d_{\beta}\left(t^{\prime}\right)\right\}\right\rangle$, for $\alpha, \beta=0,1,2,3$ using equations (3) and (4). In the absence of applied field these Green's functions are diagonal $\left(\propto \delta_{\alpha, \beta}\right)$, and due to the $\mathrm{O}(3)$ symmetry those corresponding to $\alpha=1,2,3$ are equal. We can deduce the corresponding spectral densities, $\rho_{\alpha}(\omega)$, using equation (25). The results for these spectra for $V_{0}=0$, the non-Fermi-liquid case, are shown in figures 1-4 for a range of values of the interaction $U$, and corresponding results for $V_{0} \neq 0$ are shown in figures 8-10.

In figure 1 the spectral density for the uncoupled $\alpha=0$ Majorana fermion (scalar) is shown together with those for the $\alpha=1,2,3$ Majorana fermions (vector) which are hybridized with the conduction electrons (and which are all identical). There is an additional delta function contribution to the $\alpha=0$ spectral density which is not shown but its weight as a function of $U$ is shown in figure 6 . What is clear is the cusp-like peak in the spectral density of the scalar Majorana fermion at Fermi-level and the fact that the height of this peak is $U$ dependent. The spectral density for the vector Majorana fermions also has a cusp-like peak at the Fermi-level but, in contrast to the scalar case, its value at the Fermi level is independent of $U$. In figure 2 the spectral densities of the scalar $(\alpha=0)$ Majorana fermion are shown on their own with an inset showing the linear behaviour in the neighbourhood of the Fermi level. The corresponding plots for the vector Majorana fermions are given in figure 3, which also show linear behaviour in the neighbourhood of the Fermi-level.

We can analyze these results using the renormalized perturbation theory developed in earlier work 8 . The perturbation theory is in terms of renormalized propagators for the Majorana fermions, and renormalized parameters, $\bar{\Delta}=\bar{z} \Delta, \bar{\Delta}_{0}=\bar{z}_{0} \Delta_{0}$, and $\bar{U}=\sqrt{\bar{z}_{0} \bar{z}^{3}} \Gamma_{0123}(0,0,0,0)$, where $\bar{z}, \bar{z}_{0}$, are the wavefunction renormalization factors derived from the non-singular contributions to the self energies, $\Sigma(\omega)$ and $\Sigma_{0}(\omega)$, associated with the $\alpha=1,2,3$ and $\alpha=0$ Majorana fermions respectively, $\Delta_{\alpha}(\omega)=\pi V_{\alpha}^{2} \rho_{0}(\omega)$ where $\rho_{0}(\omega)$ is the density of states of the conduction electrons, and $\Gamma_{0123}\left(\omega_{1}, \omega_{2}, \omega_{3}, \omega_{4}\right)$, is the irreducible four vertex for the Majorana interaction. The retarded Green's function for the Majorana fermions then has the form,

$$
G_{\alpha \alpha}(\omega)=\frac{\bar{z}_{\alpha}}{\omega+i \bar{\Delta}_{\alpha}-\bar{\Sigma}_{\alpha \alpha}^{(r)}(\omega)-\bar{\Sigma}_{\alpha \alpha}^{(s)}(\omega)}
$$

where $\bar{\Sigma}_{\alpha}^{(r)}(\omega)$ and $\bar{\Sigma}_{\alpha}^{(s)}(\omega)$ are the regular and singular renormalized self-energies, and we have taken the wide band limit with $\Delta_{\alpha}(\omega)$ independent of $\omega$. In the renormalized perturbation expansion there are counterterms which have to be included to satisfy the renormalization conditions and the Majorana fields are rescaled to absorb the wavefunction renormalization factors so that the free propagators in the expansion are $1 /\left(\omega+i \bar{\Delta}_{\alpha}\right)$ (seed for further details).

For the non-Fermi liquid case $V_{0}=0$ the leading order low frequency correction to the renormalized self-energy $\bar{\Sigma}(\omega)$ for the $\alpha=1,2,3$ Majorana fermions comes from the second order diagram for the singular part $\bar{\Sigma}^{(s)}(\omega)$ shown in figure $5(\mathrm{a})$. The result is

$$
\operatorname{Re} \bar{\Sigma}^{(s)}(\omega)=\left(\frac{\bar{U}}{\pi \bar{\Delta}}\right)^{2} \omega \ln \left(\frac{\omega}{\bar{\Delta}}\right)
$$

and 


$$
\operatorname{Im} \bar{\Sigma}^{(s)}(\omega)=-\frac{\pi}{2}\left(\frac{\bar{U}}{\pi \bar{\Delta}}\right)^{2} \frac{|\omega|}{\bar{\Delta}}
$$

for $T=0$. As $U \rightarrow 0, \bar{\Delta}_{\alpha} \rightarrow \Delta_{\alpha}, \bar{U} \rightarrow U$ and this result goes over to that of the standard second order perturbation theory. For the density of states of the $\alpha=1,2,3$ Majorana fermions in the neighbourhood of the Fermi level we find,

$$
\rho_{\alpha}(\omega)=\frac{1}{\pi \Delta}\left[1-\frac{\pi}{2}\left(\frac{\bar{U}}{\pi \bar{\Delta}}\right)^{2}|\omega|+\mathrm{O}\left(\omega^{2} \ln ^{2}(\omega)\right)\right],
$$

for $\alpha=1,2,3$. We see from this result that the density of states at the Fermi level is $1 / \pi \Delta$, independent of $U$, which is the same result as for the $\mathrm{O}(4)$ Anderson model, and that the leading correction term is linear in $|\omega|$. These results explain the features seen in the plots of the spectral densities of the vector Majorana shown in figures 1 and 3 . The linear term in the spectral density at the Fermi level is seen clearly and the value at the Fermi-level agrees with the theoretical value $1 / \pi \Delta$ to within $2 \%$.

The second order contribution to the renormalized self energy $\bar{\Sigma}_{0}(\omega)$ for the $\alpha=0$ Majorana fermion is the same as the for the $\mathrm{O}(4)$ model as it only involves explicitly the propagators of the renormalized $\alpha=1,2,3$ Majorana fermions (see figure 5(b)). To lowest order the result for the imaginary part is

$$
\operatorname{Im} \bar{\Sigma}_{0}(\omega)=-\frac{\bar{\Delta}}{2}\left(\frac{\bar{U}}{\pi \bar{\Delta}}\right)^{2}\left(\frac{\omega}{\bar{\Delta}}\right)^{2}
$$

for $T=0$. The leading term for the real part of the self-energy is linear in $\omega$ but is cancelled by the counterterm contribution to comply with the renormalization conditions. When this is inserted into equation (26) then we find for the corresponding spectral density

$$
\rho_{0}(\omega)=z_{0} \delta(\omega)+\frac{z_{0}}{2 \pi \bar{\Delta}}\left(\frac{\bar{U}}{\pi \bar{\Delta}}\right)^{2}+\mathrm{O}\left(\omega^{2}\right),
$$

The delta function contribution at zero frequency reflects the fact that the zero mode associated with the uncoupled $\alpha=0$ local Majorana fermion exists down to $T=0$, and it is this mode that leads to the anomalous entropy of $\ln (2) / 2$. Its weight in the spectral density is reduced to $z_{0}$, which we cannot calculate within the renormalized perturbation approach. However, we can calculate it to leading order for small $U / \pi \Delta$, and the result is $z_{0}=1 /\left(1+(U / \pi \Delta)^{2}\right)$. For large $U / \pi \Delta$, the model can be related to the two channel Kondo model and there is only one low energy scale, which is the Kondo temperature. We expect, therefore, in this limit that $z_{0} \sim T_{\mathrm{K}} / \Delta$ which implies that for large $U / \pi \Delta$, that $z_{0}$ should tend to zero exponentially in the variable $U / \pi \Delta$. This behaviour is consistent with the NRG results for the delta function weight plotted as a function of $U / \pi \Delta$ shown in figure 6 . The non-delta function contribution has a finite limit as $\omega \rightarrow 0$ given in renormalized perturbation theory by the the second term in equation (31). Unlike the spectra for the $\alpha=1,2,3$ Majorana fermions the value of this contribution at the Fermi level depends on $U$ and monotonically increases with increase of $U / \pi \Delta$. We see from equation (31) that to lowest order in $U / \pi \Delta$ it is proportional to $U^{2} /(\pi \Delta)^{3}$. As the second order diagram in the renormalized perturbation theory gives the exact result to order $\omega^{2}$ for $\operatorname{Im} \bar{\Sigma}_{0}(\omega)$ there should be no further contributions to the leading two terms in equation (31). In the large $U$ limit we deduced from a comparison of the results with those for the two channel Kondo model (seet) that $\bar{U} / \pi \bar{\Delta} \rightarrow 1$. The height of the peak at the Fermi-level compared with that for the vector term in this limit is then given by $\bar{z}_{0} / 2 \bar{z}$ (using $\bar{\Delta}=\bar{z} \Delta$ ), and provides an estimate of the ratio of the wavefunction renormalization factors of the scalar and vector Majorana fermions.

The correction terms in (31) arise from terms in the imaginary part of the self-energy which behave as $\omega^{4}$ for small $\omega$. The second order diagram in the renormalized perturbation theory gives an contribution $\omega^{4}$ but there are also contributions to this order from higher order diagrams. More important, however, are contributions to the imaginary part which behave like $\omega^{2}|\omega|$, as they result in corrections to (31) which behave like $|\omega|$. Such a term is generated from the higher order diagram shown in figure 7 . The asymptotic contribution to the spectral density from this diagram as $\omega \rightarrow 0$ is

$$
-2 z_{0} \pi^{2}\left(\frac{\bar{U}}{\pi \bar{\Delta}}\right)^{4} \frac{|\omega|}{\bar{\Delta}^{2}}
$$

There may be other higher order diagrams which contribute terms to this order $\omega$ but checking this is difficult. The explicit evaluation of the higher order diagrams is in general complicated and there does not seem to be any simple way of seeing whether or not a diagram gives a contribution of this order. It is clear from the results shown in figure 2 
that there is a net contribution of this order as there is clear $|\omega|$ dependence in the neighbourhood of the Fermi level. If $U$ is small compared to $\pi \Delta$ then the second order diagram which gives an $\omega^{2}$ correction to (31) should dominate over the leading $|\omega|$ term which is of fourth order. The second order result corresponding to (31) is

$$
\rho_{0}(\omega)=\left(1-\left(\frac{U}{\pi \Delta}\right)^{2}\right) \delta(\omega)+\frac{1}{2 \pi \Delta}\left(\frac{U}{\pi \Delta}\right)^{2}\left(1-\frac{\omega^{2}}{2 \Delta^{2}}\right)+. .
$$

The $\omega^{2}$ behaviour of $\rho_{0}(\omega)$ in the neighbourhood of the Fermi level in the small U regime is evident in figure 4 which gives a plot of $\rho_{0}(\omega)$ for $U / \pi \Delta=0.01$.

For $V_{0} \neq 0$ the zero Majorana fermion is no longer uncoupled from the conduction electrons and for $U=0$ the corresponding spectral density has a resonance width of $\bar{\Delta}_{0}$. Having lost the zero mode in this channel the scattering the vector Majorana fermions with the scalar Majorana fermion with the interaction $U$ is no longer singular. For $\bar{\Delta}_{0} / \bar{\Delta} \ll 1$, the resonance in the scalar channel is very narrow compared with that of the vector channel, and there is a low energy range in which the behaviour is very similar to that found in the marginal case. There is a cross-over eventually on reducing the energy scale to a Fermi-liquid form of behaviour. This is apparent in the plots of the spectra in figures 8-10 for the case $\Delta_{0} / \Delta=10^{-4}$. In figure 8 the spectral densities of both the scalar and vector Majorana fermions are shown for the same set of values for $U / \pi \Delta$ as in figure 1. Due to the small value which was used for the ratio $\Delta_{0} / \Delta$ the spectra for the vector Majorana fermions shown in figure 9 are quite similar to the corresponding curves for the marginal Fermi-liquid case shown in figure 3. The difference is only revealed when we compare the insets in the two figures. On the very low energy scale around the Fermi level there is a distinct flattening and rounding of the spectrum seen in the inset in figure 9 , consistent with an $\omega^{2}$ behaviour in contrast to the form $|\omega|$ seen in the inset in figure 3 (which persisted down to the lowest energy scales for which the spectra were calculated).

If we apply second order perturbation theory for finite $\Delta_{0}$ then, in the low frequency range $\omega \ll \Delta$, the imaginary part of the self-energy

has the form,

$$
\operatorname{Im} \Sigma(\omega)=\left(\frac{U}{\pi \Delta}\right)^{2}\left[-\omega \tan ^{-1}\left(\frac{\omega}{\Delta_{0}}\right)+\frac{\Delta_{0}}{2} \ln \left(1+\frac{\omega^{2}}{\Delta_{0}^{2}}\right)\right] .
$$

In the range $\omega>\Delta_{0}$ this behaves essentially as $|\omega|$, as in the non-Fermi liquid case (29). Only in the very low frequency range $\omega \ll \Delta_{0}$ do we get the influence of the Fermi-liquid fixed point and the $\omega^{2}$ behaviour. In the low frequency limit $\omega \ll \Delta_{0}$, the spectral density of the vector Majorana fermions is then given by

$$
\rho_{\alpha}(\omega)=\frac{1}{\pi \Delta}\left[1-\frac{\omega^{2}}{2 \Delta \Delta_{0}}\left\{\frac{2 \Delta_{0}}{\Delta}+\left(1+\frac{4 \Delta_{0}}{\Delta}\right)\left(\frac{U}{\pi \Delta}\right)^{2}\right\}\right],
$$

for $\alpha=1,2,3$. For large values of $U$ we can apply the renormalized perturbation approach and the corresponding result for the spectrum of the vector Majorana fermions in the very low frequency limit $\omega \ll \bar{\Delta}_{0}$ is

$$
\rho_{\alpha}(\omega)=\frac{1}{\pi \Delta}\left[1-\frac{\omega^{2}}{2 \bar{\Delta} \bar{\Delta}_{0}}\left\{\frac{2 \bar{\Delta}_{0}}{\bar{\Delta}}+\left(\frac{\bar{U}}{\pi \bar{\Delta}}\right)^{2}\right\}\right] .
$$

The spectra for the scalar Majorana fermion for $\Delta_{0} \neq 0$, however, are very different from those for $\Delta_{0}=0$ as can been seen by comparing the results given in figure 10 with those in figure 2 . There is no zero mode and the peaks at the Fermi level coincide. In the very low frequency range $\omega \ll \bar{\Delta}_{0}$ we find for $\rho_{0}(\omega)$ using the renormalized perturbation theory the result,

$$
\rho_{0}(\omega)=\frac{1}{\pi \Delta_{0}}\left[1-\frac{\omega^{2}}{2 \bar{\Delta}_{0}^{2}}\left\{2+\frac{\bar{\Delta}_{0}}{\bar{\Delta}}\left(\frac{\bar{U}}{\pi \bar{\Delta}}\right)^{2}\right\}\right] .
$$

The value of the density of states at the Fermi level for the results shown in figure 10 are within $2 \%$ of the Freidel sum rule result $1 / \pi \Delta_{0}$ for all values of $U$. The $\omega^{2}$ behaviour in the very low energy region near the Fermi-level is clear in the inset in figure 10. 


\section{B. Dynamic spin and charge susceptibilities}

We use the same approach to calculate the two particle Green's functions corresponding to the dynamic spin and charge susceptibilities. The corresponding spectral densities are of the form

$$
\begin{aligned}
A_{Q}(\omega)= & \frac{1}{Z} \sum_{n m}|\langle n|Q| m\rangle|^{2} \delta\left(\omega-\left(E_{n}-E_{m}\right)\right) \\
& \times\left(e^{-\beta E_{m}}-e^{-\beta E_{n}}\right)
\end{aligned}
$$

with $Q=g \mu_{\mathrm{B}}\left(n_{d, \uparrow}-n_{d, \downarrow}\right) / 2$ for spin and $Q=\left(n_{d, \uparrow}+n_{d, \downarrow}-1\right) / 2$ for charge. This is similar in form to that for

the single particle Green's function given in equation (25), which corresponds to $Q=c_{d \sigma}^{\dagger}$ with a difference in sign in the factor in the last bracket due to the use of the commutator rather than the anticommutator for the spin and charge Green's functions. Using the procedure outlined in the appendix these can be expressed in terms of reduced matrix elements and the degeneracy with respect to $T_{z}$ can be explicitly summed over (see equation (51)). Both the operators for spin and charge transform as irreducible tensors of the form $Q_{0}^{1}$, and the result in each case gives

$$
\begin{aligned}
A_{Q}(\omega)= & \frac{1}{3 Z} \sum_{n m}|\langle n|| Q|| m\rangle|^{2} \delta\left(\omega-\left(E_{n}-E_{m}\right)\right) \\
& \left(e^{-\beta E_{m}}-e^{-\beta E_{n}}\right)
\end{aligned}
$$

where $\langle n\|Q\| m\rangle$ is the reduced matrix element (see equation (51)).

The dynamic susceptibilities for the non-interacting model $(U=0)$ can be evaluated analytically. There are two parts to the $d$ contribution to the spin susceptibility $\chi_{\mathrm{s}}(\omega)$. One arises from the bubble diagram, figure 11 (a), where the propagators involved are those corresponding to a component of the vector Majorana fermion which we denote by $\chi_{\mathrm{d}}^{\mathrm{vv}}(\omega)$ (in which we absorb factors of $g \mu_{\mathrm{B}}$ ). This contribution is just half of that obtained for the standard symmetric Anderson model with the real part given by

$$
\operatorname{Re} \chi_{\mathrm{d}}^{\mathrm{vv}}=\frac{\Delta / 4 \pi}{\left(\omega^{2}+4 \Delta^{2}\right)}\left\{\ln \left|\frac{\Delta^{2}}{\omega^{2}+\Delta^{2}}\right|+\frac{4 \Delta}{\omega} \tan ^{-1}\left(\frac{\omega}{\Delta}\right)\right\}
$$

and the imaginary part by

$$
\operatorname{Im} \chi_{d}^{\mathrm{vv}}(\omega)=\frac{\Delta / 2 \pi}{\left(\omega^{2}+4 \Delta^{2}\right)}\left\{\frac{\Delta}{\omega} \ln \left(1+\frac{\omega^{2}}{\Delta^{2}}\right)+\tan ^{-1}\left(\frac{\omega}{\Delta}\right)\right\}
$$

The other contribution, which we denote by $\chi_{\mathrm{d}}^{\mathrm{vs}}(\omega)$, is from bubble diagram, figure 11(b), in which one of the propagators corresponds to a component of the vector Majorana fermion while the other propagator is for the scalar Majorana fermion. The real part is given by

$$
\begin{aligned}
\operatorname{Re} \chi_{\mathrm{d}}^{\mathrm{vs}}(\omega) & =\frac{\omega^{2} \Delta / 4 \pi}{\left(\omega^{2}+\Delta_{0}^{2}-\Delta^{2}\right)^{2}+4 \omega^{2} \Delta^{2}} \\
& \times\left\{\left(\frac{\omega^{2}-\Delta_{0}^{2}+\Delta^{2}}{2 \omega^{2}}\right) \ln \left|\frac{\Delta^{2}}{\omega^{2}+\Delta_{0}^{2}}\right|\right. \\
& \left.+\frac{2 \Delta_{0}}{\omega} \tan ^{-1}\left(\frac{\omega}{\Delta_{0}}\right)\right\} \\
& +\left\{\text { similar term with } \Delta_{0} \leftrightarrow \Delta\right\},
\end{aligned}
$$

and the imaginary part by

$$
\begin{aligned}
\operatorname{Im} \chi_{\mathrm{d}}^{\mathrm{vs}}(\omega) & =\frac{\omega \Delta \Delta_{0} / 4 \pi}{\left(\omega^{2}+\Delta_{0}^{2}-\Delta^{2}\right)^{2}+4 \omega^{2} \Delta^{2}} \\
& \times\left\{\left(\frac{\omega^{2}-\Delta_{0}^{2}+\Delta^{2}}{\omega \Delta_{0}}\right) \tan ^{-1}\left(\frac{\omega}{\Delta_{0}}\right)\right. \\
& +\ln \left|\frac{\left(\omega^{2}+\Delta^{2}\right)}{\Delta_{0}^{2}}\right| \\
& \left.+ \text { similar terms with } \Delta_{0} \leftrightarrow \Delta\right\},
\end{aligned}
$$


In the limit $\Delta_{0} \rightarrow \Delta$ the vector-scalar contribution is the same as the vector-vector contribution and we recover the results for the dynamic susceptibility of the symmetric Anderson model. In the limit $\Delta_{0} \rightarrow 0$, where we have a zero mode, the real part of the vector-scalar contribution develops a logarithmic singularity,

$$
\operatorname{Re} \chi_{\mathrm{d}}^{\mathrm{vs}}(\omega)=\frac{\Delta}{4 \pi\left(\omega^{2}+\Delta^{2}\right)} \ln \left|\frac{\Delta}{\omega}\right|,
$$

and the imaginary part has a discontinuity at the origin,

$$
\operatorname{Im} \chi_{d}^{\mathrm{vs}}(\omega)=\frac{\operatorname{sgn}(\omega) \Delta}{8\left(\omega^{2}+\Delta^{2}\right)} .
$$

We also have some exact reults for $U \neq 0$ from the Shiba relation which relates the imaginary part of $\chi_{\mathrm{s}}(\omega) / \omega$ for the impurity to the real part of $\chi_{\mathbf{s}}(\omega)$ in the limit $\omega \rightarrow 0$,

$$
\lim _{\omega \rightarrow 0}\left\{\frac{\operatorname{Im} \chi_{\mathrm{s}}(\omega+i \delta)}{2 \pi \omega}\right\}=\frac{\left(\operatorname{Re} \chi_{\mathrm{s}}(0)\right)^{2}}{\left(g \mu_{\mathrm{B}}\right)^{2}} .
$$

This result, however, is only applicable to the standard symmetric Anderson model where $\Delta_{0}=\Delta$.

We can use the exact results we have for this model to gauge the accuracy of our NRG calculations. In figure 12 we plot the imaginary part of $\chi_{s}(\omega)$ divided by the square of the real part for various values of $U$. According to the Shiba relation these should all pass through the same point at $\omega=0$ and this is clearly seen in the results figure 12 . The actual value at this point is about $8 \%$ too high, reflecting errors of a few $\%$ in the individual susceptibilities. The overall accuracy over the full frequency range can be gauged by a comparison of the NRG results with the exact results deduced from equations (40), (41), (42) and (43) for $U=0$. In figure 13 we plot the NRG results for the real part of $\chi_{\mathrm{s}}(\omega)$ for various values of $\Delta_{0}$, and compare them with the analytic results. It can be seen that the two sets of results are in good agreement over the complete frequency range.

To see how the dynamic spin susceptibility for finite $U$ differs from that for the standard symmetric Anderson model we have plotted both the imaginary part and real part in figures 14 and 15 for $U / \pi \Delta=1.5$ and various values of $\Delta_{0}$. As we reduce $\Delta_{0}$ the imaginary part changes more and more rapidly at $\omega=0$ until finally for $\Delta_{0}=0$ it has a finite discontinuous jump at this point as can be in figure 16, where the results for $\Delta_{0}=0$ are shown for various values of $U$. The peak in real part of the spin susceptibility narrows considerably on reducing $\Delta_{0}$ as can be seen in figure 15. To be able to compare the results for various values of $\Delta_{0}$ the values shown in figure 15 have been multiplied by the factor $1 / \chi_{\mathrm{s}}(0)$, so it is not so apparent that the narrowing is accompanied by a sharp increase in the absolute value at low frequencies. In the limit $\Delta_{0}=0$ the real part of $\chi_{\mathrm{s}}(\omega)$ has a singularity at $\omega=0$. A discontinuity in the imaginary part of $\chi_{s}(\omega)$ for $\Delta_{0}=0$ at $\omega=0$ with a logarithmic singularity in the real part is predicted from equations (45) and (44) for $U=0$, so it is not surprising that these features persist for small values of $U$. For large $U$ we can make predictions from our results about the behaviour of the spin susceptibility of the corresponding two channel Kondo model over the full frequency range. We look at this in detail in the next section.

\section{SPIN DYNAMICS OF THE TWO CHANNEL KONDO MODEL}

In the large $U$ regime we can apply the mapping the $\mathrm{O}(3)$ Anderson model in the localized limit and the NRG results of previous section to deduce the spin dynamics of the two channel Kondo model over the complete frequency range. As the mapping is for the spin degrees of freedom of the TCKM, which is the only part with an interaction term, it cannot be used to calculate all the relevant properties of the TCKM, only those where the other degrees of freedom of the conduction electrons do not contribute. It can be used for example to deduce the the impurity contribution to the thermodyamics, such as susceptibility and specific heat, and also for the spin dynamics of the impurity, where the uncoupled degrees of freedom factorize and cancel out. This is a distinct advantage for NRG calculations to use the equivalent in one channel model because a larger percentage of the states can be retained after each diagonalization in the iterative procedure. The number of states that can retained be at each stage is proportional to $1 / 4^{N_{c}}$, where $N_{c}$ is the number of channels, so this can be serious limitation when the number of channels is increased. However, quite apart from these practical considerations, the relation between the TCKM and the the $\mathrm{O}(3)$ Anderson model provides a simple way of understanding in terms of renormalized Majorana fermions 1 te.

We saw in the previous section that the real part of the dynamic spin susceptibility $\left(\Delta_{0}=0\right)$ at $T=0$ has a logarithmic singularity in $\omega$ for $U=0$ and that in the NRG results this singular behaviour persists for finite $U_{\text {S }}^{\text {Such }}$ a term in $\ln \omega$ has also been found in direct NRG calculations of the dynamic susceptibility for the TCKM 23. To 
analyse the situation in the large $U$ limit to use the mapping to the TCKM it is useful to work with a conserved quantity, which in this case is not the spin but $\mathbf{T}$ the spin plus isospin. The corresponding local dynamic susceptibility we denote by $\chi_{\mathbf{t}}(\omega)$, where $\mathbf{t}=\sigma_{d}+\tau_{d}$. Because the charge fluctuations of the impurity are suppressed for large $U$ this susceptibility becomes identical with the spin susceptibility in the large $U$ limit. We can use the NRG approach described in the last section to calculate $\chi_{\mathbf{t}}(\omega)$ and verify this explicitly. We find for $U / \pi \Delta=4$ at the most a $5 \%$ difference between $\chi_{\mathbf{t}}(\omega)$ and $\chi_{\mathbf{s}}(\omega)$ over the complete low frequency range $\omega \ll U$.

For the large $U$ regime we can apply our renormalized perturbation approach to calculate the $\omega$ dependence of $\chi_{\mathbf{t}}(\omega)$, following the same steps we used in our earlier work to calculate its temperature dependence in the zero frequency limit 1 . We first calculate the contribution due to the non-interacting renormalized Majorana fermions. This is given by the vector-vector bubble shown in figure 11 (a), together with an overall factor of 4 and $\Delta$ replaced by $\bar{\Delta}$ so that for $T=0$,

$$
\operatorname{Re} \chi_{\mathbf{t}}(\omega)=\frac{\bar{\Delta}}{\pi\left(\omega^{2}+4 \bar{\Delta}^{2}\right)}\left\{\ln \left|\frac{\bar{\Delta}^{2}}{\omega^{2}+\bar{\Delta}^{2}}\right|+\frac{4 \bar{\Delta}}{\omega} \tan ^{-1}\left(\frac{\omega}{\bar{\Delta}}\right)\right\} .
$$

Because the single particle Green's function and the quasiparticle Green's function in the renormalized perturbation approach differ by a wavefunction renormalization factor $\bar{z}$, one might have expected an extra $\bar{z}^{2}$ multiplying (477) to give the two particle Green's function corresponding to the dynamic susceptibility. This is not the case because there is a compensating factor associated with the coupling to the external field. The net result is that these two terms cancel so that in the quasiparticle Hamiltonian in the presence of a external field the quasi-particles are coupled to the field with the same coupling as the bare particles, i.e. there is no g-factor renormalization. In the zero frequency limit (47) gives $\chi_{\mathbf{t}}(0)=1 / \pi \bar{\Delta}$, which corresponds to zero temperature impurity contribution to the total susceptibility we found in our earlier work in the same limit. The leading order correction term to this result arises from a second order term in $\bar{U}$, shown in figure 17, where the scalar-vector bubble shown in figure 11, gives a $\ln \omega$ contribution similar to the $\ln T$ dependence in the same susceptibility at $\omega=0$ seet, so that asymptotically as $\omega \rightarrow 0$ we find

$$
\operatorname{Re} \chi_{\mathbf{t}}(\omega)=\left(\frac{1}{\pi \bar{\Delta}}-\left(\frac{\bar{U}}{\pi \bar{\Delta}}\right)^{2} \frac{\ln (\omega / \bar{\Delta})}{\pi \bar{\Delta}}\right) .
$$

The correspondence of the calculation of the $\ln \omega$ terms with the calculation of the $\ln T$ terms for the zero frequency susceptibility is so precise that we can appeal to arguments in our earlier work $⿴$ to show that higher order $\ln \omega$ terms cancel in the same way as the higher order $\ln T$ terms.

Though we cannot explicitly carry out the full renormalization programme to calculate the renormalized parameters $\bar{U}$ and $\bar{\Delta}$ in terms of the bare parameters $U$ and $\Delta$, we can use the results $\bar{U} / \pi \bar{\Delta}=1, \pi \bar{\Delta}=T_{\mathrm{K}}$ from our earlier work, which were obtained by equating the expressions for the temperature dependence of the susceptibility in the large $U$ limit. We then find for the asymptotic behaviour of the dynamic susceptibility of the two channel Kondo model $\chi_{\mathrm{KM}}(\omega)$ as $\omega \rightarrow 0, \omega=0$ seet, so that asymptotically as $\omega \rightarrow 0$ we find

$$
\operatorname{Re} \chi_{\mathrm{KM}}(\omega)=\frac{1}{T_{\mathrm{K}}}\left(1-\ln \left(\omega \pi / T_{\mathrm{K}}\right)\right) .
$$

In figure 18 we plot the real part of both $\chi_{\mathbf{t}}(\omega)$ and $\chi_{\mathrm{s}}(\omega)$ versus $\ln (\omega)$ for $U / \pi \Delta=4$ and the asymptotic form given in equation (49) is clearly seen in the low frequency limit. We have also calculated $T_{\mathrm{K}}$ both from the limiting form of $\chi_{\mathbf{t}}(T)$ as $T \rightarrow 0$ and $\chi_{\mathbf{t}}(\omega)$ as $\omega \rightarrow 0$, for $U / \pi \Delta=4$ and $V=0.00141$ and find $T_{\mathrm{K}}=1.3 \pm 0.2$ and $T_{\mathrm{K}}=1.28 \pm 0.1$, respectively, which confirms the result (49) to within the error limits of the calculations.

\section{CONCLUSIONS}

We have shown that $\mathrm{O}(3)$ Anderson model in the large $U$ limit can describe precisely the interacting spin degrees of freedom of the linear dispersion two channel Kondo model. This extends earlier work in that it establishes that this relationship applies over the full energy range of the two channel model and not just in the immediate region of the low energy fixed point. It enables us to calculate the impurity contribution to the thermodynamics, and also the impurity spin dynamics, for the TCKM from the $\mathrm{O}(3)$ Anderson model in the strong correlation regime. This has a number of advantages. The $\mathrm{O}(3)$ model is most conveniently expressed in terms of Majorana fermions and the low energy fixed point of this model corresponds to free Majorana fermions whatever the value of the on-site interaction $U$, so the nature of the fixed point is the same in weak and strong coupling. This is similar to the standard Anderson model, which has a Fermi liquid fixed point, independent of the value of $U$. The main difference between 
the $\mathrm{O}(3)$ and the standard Anderson model, is that $\mathrm{O}(3)$ model has a zero mode in the case that corresponds to the channel isotropic TCKM which results in singular scattering of the renormalized Majorana fermions giving rise to logarithmically divergent contributions to the impurity specific heat coefficient, susceptibility, and dynamic spin-spin response function. This singular low energy behaviour corresponds the marginal Fermi liquid theory that mas put forward to describe the anomalous behaviour observed in the normal state of the cuprate superconductors24. The description of the TCKM in terms of Majorana fermions not only gives a simple interpretation of the many-body excitations at the low energy fixed point, both for the channel isotropic and anisotropic cases, but can also be applied on all energy scales.

To find the renormalizations of the Majorana fermions explicitly we use the NRG approach to calculate the Majorana spectral densities. We have calculated weight of the zero Majorana fermion mode and have shown that it decreases exponentially with $U$ in the large $U$ regime. We have also demonstrated the $|\omega|$ behaviour of the other Majorana fermion modes in the marginal Fermi liquid case as $\omega \rightarrow 0$. We have used the same approach to calculate the two particle response functions which we have determined over the full frequency range for $T=0$. We have exploited the mapping between the models to deduce the local dynamic spin susceptibility of the TCKM. Renormalized perturbation theory has been used to interpret the results and to calculate the coefficient of the $\ln \omega$ term in asymptotic form of the real part of the dynamic spin susceptibility of the TCKM in the limit $\omega \rightarrow 0$.

\section{Acknowledgment}

We are grateful for the support of an EPSRC research grant (GR/J85349), and for a DFG research grant (Bu965$1 / 1$ ) for one of us (RB).

\section{APPENDIX}

Here we outline the steps involved in the evaluation of the spectral density given in equation (25). The many-body states $|n\rangle$ are classified using the quantum numbers $T$ and $T_{z}$ of the total angular momentum (spin plus isospin) and expressed in the form $\left|T_{n}, T_{z, n}, w_{n}\right\rangle$, where $w_{n}$ labels any further degeneracies of the state $|n\rangle$. The degeneracies associated with the z-component of angular momentum in (25) can be summed over explicitly. The creation and

annihilation operators, $c_{d, \sigma}^{\dagger}$ and $c_{d, \sigma}^{\dagger}$, first of all have to be expressed in terms of the irreducible tensor operators $V_{q}^{k}$, $\mathrm{k}=1,0, \mathrm{q}=1,0,-1$. From equation $(32)$ in reference 3 ,

$$
\begin{gathered}
V_{0}^{0}=\frac{1}{\sqrt{2}}\left(c_{d, \downarrow}+c_{d, \downarrow}^{\dagger}\right) \\
V_{1}^{1}=c_{d, \uparrow}^{\dagger} \\
V_{0}^{1}=\frac{1}{\sqrt{2}}\left(c_{d, \downarrow}-c_{d, \downarrow}^{\dagger}\right) \\
V_{-1}^{1}=-c_{d, \uparrow}
\end{gathered}
$$

Then the Wigner-Eckart Theorem,

$$
\begin{gathered}
\left\langle T^{\prime}, T_{z}^{\prime}, w^{\prime}\left|V_{q}^{k}\right| T, T_{z}, w\right\rangle=\frac{1}{\sqrt{2 T^{\prime}+1}}\left\langle T^{\prime}, w^{\prime}|| V_{q}^{k}|| T, w\right\rangle \\
\times\left\langle T, T_{z}, k, q \mid T^{\prime}, T_{z}^{\prime},\right\rangle
\end{gathered}
$$

is used to express all the matrix elements in terms of the reduced matrix elements $\left\langle T^{\prime}, w^{\prime} \| V_{q}^{k}|| T, w\right\rangle$ which are independent of the $T_{z}$ quantum numbers, where $\left\langle T, T_{z}, k, q \mid T^{\prime}, T_{z}^{\prime},\right\rangle$ are the Clebsch-Gordon coefficients. For example for the spin down matrix elements we find

$$
\begin{aligned}
\left|\left\langle n\left|c_{d \downarrow}^{\dagger}\right| m\right\rangle\right|^{2} & =\left|\left\langle T_{n}, T_{z, n}, w_{n}\left|c_{d \downarrow}^{\dagger}\right| T_{m}, T_{z, m}, w_{m}\right\rangle\right|^{2} \\
& =\left|\left\langle T_{n}, T_{z, n}, w_{n}\left|\frac{1}{\sqrt{2}}\left(V_{0}^{1}-V_{0}^{0}\right)\right| T_{m}, T_{z, m}, w_{m}\right\rangle\right|^{2}
\end{aligned}
$$




$$
\begin{aligned}
= & \frac{1}{2\left(2 T_{n}+1\right)} \mid\left\langle T_{n}, w_{n} \| V_{0}^{1}|| T_{m}, w_{m}\right\rangle\left\langle T_{m}, T_{z, m}, 1,0 \mid T_{n}, T_{z, n}\right\rangle \\
& -\left.\left\langle T_{n}, w_{n}|| V_{0}^{0}|| T_{m}, w_{m}\right\rangle \delta_{T_{n}, T_{m}} \delta_{T_{z, n}, T_{z, m}}\right|^{2}
\end{aligned}
$$

For $T_{n} \neq T_{m}$, the $V_{0}^{0}$-term does not contribute and for this term we find

$$
\begin{aligned}
\sum_{T_{z}}\left|\left\langle n\left|c_{d \downarrow}^{\dagger}\right| m\right\rangle\right|^{2} & =\frac{1}{2\left(2 T_{n}+1\right)}\left|\left\langle T_{n}, w_{n}|| V_{0}^{1}|| T_{m}, w_{m}\right\rangle\right|^{2} \sum_{T_{z}}\left|\left\langle T_{m}, T_{z}, 1,0 \mid T_{n}, T_{z}\right\rangle\right|^{2} \\
& =\frac{1}{6}\left|\left\langle T_{n}, w_{n}|| V_{0}^{1}|| T_{m}, w_{m}\right\rangle\right|^{2}
\end{aligned}
$$

For the case $T_{n}=T_{m}=T$ we find

$$
\sum_{T_{z}}\left|\left\langle n\left|c_{d \downarrow}^{\dagger}\right| m\right\rangle\right|^{2}=\frac{1}{6}\left|\left\langle T, w_{n}|| V_{0}^{1}|| T, w_{m}\right\rangle\right|^{2}+\frac{1}{2}\left|\left\langle T, w_{n} \|\left|V_{0}^{0}\right| \mid T, w_{m}\right\rangle\right|^{2}
$$

The reduced matrix elements $\left\langle T_{n}, w_{n} \| V_{0}^{q}|| T_{m}, w_{m}\right\rangle$ are calculated from those of the previous iteration in two steps. We start from the expression for the reduced matrix element with respect to the eigenstates of the impurity-conduction electron chain with $N+1$ sites,

$$
\begin{array}{r}
\left\langle T_{n}, w_{n}\left\|V_{0}^{q}\right\| T_{m}, w_{m}\right\rangle_{N+1}=\sum_{r_{n}, p_{n}, r_{m}, p_{m}} U_{T_{n}}\left(w_{n}, r_{n}, p_{n}\right) U_{T_{m}}\left(w_{m}, r_{m}, p_{m}\right) \\
\times\left\langle T_{n}, r_{n}, p_{n}\left\|\left|V_{0}^{q} \| T_{m}, r_{m}, p_{m}\right\rangle_{N+1},\right.\right.
\end{array}
$$

in terms of the basis states $\left|T_{n}, r_{n}, p_{n}\right\rangle_{N+1}$ used for this calculation (these are constructed from the eigenstates of the $N$ site chain $\left|T_{n}, r_{n}\right\rangle_{N}$ and the states for the additional site labelled by $p_{n}$, see reference $\mathrm{B}$ for further details). The coefficients $U_{T_{n}}\left(w_{n}, r_{n}, p_{n}\right)$ are the components of the eigenstate $\left|T_{n}, w_{n}\right\rangle_{N+1}$ with respect to this basis. The reduced matrix elements $\left\langle T_{n}, r_{n}, p_{n} \|\left|V_{0}^{q}\right| \mid T_{m}, r_{m}, p_{m}\right\rangle_{N+1}$ have to be related to the reduced matrix elements of the previous step as follows:

$$
\begin{aligned}
\left\langle T, r, 1\left\|V_{0}^{1}\right\| T+1, r^{\prime}, 1\right\rangle_{N+1} & =\sqrt{\frac{2 T+3}{2(T+1)}}\left\langle T-\frac{1}{2}, r\left\|V_{0}^{1}\right\| T+\frac{1}{2}, r^{\prime}\right\rangle_{N} \\
\left\langle T, r, 1\left\|V_{0}^{1}\right\| T-1, r^{\prime}, 1\right\rangle_{N+1} & =\sqrt{\frac{2 T+1}{2 T}}\left\langle T-\frac{1}{2}, r\left\|V_{0}^{1}\right\| T-\frac{3}{2}, r^{\prime}\right\rangle_{N} \\
\left\langle T, r, 1\left\|V_{0}^{1}\right\| T, r^{\prime}, 1\right\rangle_{N+1} & =\frac{1}{\sqrt{2} T} \sqrt{(T+1)(2 T-1)}\left\langle T-\frac{1}{2}, r\left\|V_{0}^{1}\right\| T-\frac{1}{2}, r^{\prime}\right\rangle_{N} \\
\left\langle T, r, 1\left\|V_{0}^{0}\right\| T-1, r^{\prime}, 1\right\rangle_{N+1} & =\sqrt{\frac{2 T+1}{2 T}}\left\langle T-\frac{1}{2}, r\left\|V_{0}^{0}\right\| T-\frac{1}{2}, r^{\prime}\right\rangle_{N} \\
\left\langle T, r, 2\left\|V_{0}^{1}\right\| T+1, r^{\prime}, 2\right\rangle_{N+1} & =-\sqrt{\frac{2 T+3}{2(T+1)}}\left\langle T-\frac{1}{2}, r\left\|V_{0}^{1}\right\| T+\frac{1}{2}, r^{\prime}\right\rangle_{N} \\
\left\langle T, r, 2\left\|V_{0}^{1}\right\| T-1, r^{\prime}, 2\right\rangle_{N+1} & =-\sqrt{\frac{2 T+1}{2 T}}\left\langle T-\frac{1}{2}, r\left\|V_{0}^{1}\right\| T-\frac{3}{2}, r^{\prime}\right\rangle_{N} \\
\left\langle T, r, 2\left\|V_{0}^{1}\right\| T, r^{\prime}, 2\right\rangle_{N+1} & =-\frac{1}{\sqrt{2} T} \sqrt{(T+1)(2 T-1)}\left\langle T-\frac{1}{2}, r\left\|V_{0}^{1}\right\| T-\frac{1}{2}, r^{\prime}\right\rangle_{N} \\
\left\langle T, r, 2\left\|V_{0}^{0}\right\| T-1, r^{\prime}, 2\right\rangle_{N+1} & =-\sqrt{\frac{2 T+1}{2 T}}\left\langle T-\frac{1}{2}, r\left\|V_{0}^{0}\right\| T-\frac{1}{2}, r^{\prime}\right\rangle_{N}
\end{aligned}
$$




$$
\begin{aligned}
& \left\langle T, r, 3\left\|V_{0}^{1}\right\| T+1, r^{\prime}, 3\right\rangle_{N+1}=-\sqrt{\frac{2 T+1}{2(T+1)}}\left\langle T+\frac{1}{2}, r\left\|V_{0}^{1}\right\| T+\frac{3}{2}, r^{\prime}\right\rangle_{N} \\
& \left\langle T, r, 3\left\|V_{0}^{1}\right\| T-1, r^{\prime}, 3\right\rangle_{N+1}=-\sqrt{\frac{(T+1)(2 T-1)}{2 T(T+1)}}\left\langle T+\frac{1}{2}, r\left\|V_{0}^{1}\right\| T-\frac{1}{2}, r^{\prime}\right\rangle_{N} \\
& \left\langle T, r, 3\left\|V_{0}^{1}\right\| T, r^{\prime}, 3\right\rangle_{N+1}=-\frac{1}{\sqrt{2}(T+1)} \sqrt{T(2 T+3)}\left\langle T+\frac{1}{2}, r\left\|V_{0}^{1}\right\| T+\frac{1}{2}, r^{\prime}\right\rangle_{N} \\
& \left\langle T, r, 3\left\|V_{0}^{0}\right\| T, r^{\prime}, 3\right\rangle_{N+1}=-\sqrt{\frac{2 T+1}{2(T+1)}}\left\langle T+\frac{1}{2}, r\left\|V_{0}^{0}\right\| T+\frac{1}{2}, r^{\prime}\right\rangle_{N} \\
& \left\langle T, r, 4\left\|V_{0}^{1}\right\| T+1, r^{\prime}, 4\right\rangle_{N+1}=\sqrt{\frac{2 T+1}{2(T+1)}}\left\langle T+\frac{1}{2}, r\left\|V_{0}^{1}\right\| T+\frac{3}{2}, r^{\prime}\right\rangle_{N} \\
& \left\langle T, r, 4\left\|V_{0}^{1}\right\| T-1, r^{\prime}, 4\right\rangle_{N+1}=\sqrt{\frac{(T+1)(2 T-1)}{2 T(T+1)}}\left\langle T+\frac{1}{2}, r\left\|V_{0}^{1}\right\| T-\frac{1}{2}, r^{\prime}\right\rangle_{N} \\
& \left\langle T, r, 4\left\|V_{0}^{1}\right\| T, r^{\prime}, 4\right\rangle_{N+1}=\frac{1}{\sqrt{2}(T+1)} \sqrt{T(2 T+3)}\left\langle T+\frac{1}{2}, r\left\|V_{0}^{1}\right\| T+\frac{1}{2}, r^{\prime}\right\rangle_{N} \\
& \left\langle T, r, 4\left\|V_{0}^{0}\right\| T, r^{\prime}, 4\right\rangle_{N+1}=\sqrt{\frac{2 T+1}{2(T+1)}}\left\langle T+\frac{1}{2}, r\left\|V_{0}^{0}\right\| T+\frac{1}{2}, r^{\prime}\right\rangle_{N} \\
& \left\langle T, r, 1\left\|V_{0}^{1}\right\| T, r^{\prime}, 4\right\rangle_{N+1}=\frac{(-1)^{N+1}}{\sqrt{2 T(T+1)}}\left\langle T-\frac{1}{2}, r\left\|V_{0}^{1}\right\| T+\frac{1}{2}, r^{\prime}\right\rangle_{N} \\
& \left\langle T, r, 1\left\|V_{0}^{1}\right\| T-1, r^{\prime}, 4\right\rangle_{N+1}=\frac{(-1)^{N+1}}{\sqrt{2} T}\left\langle T-\frac{1}{2}, r\left\|V_{0}^{1}\right\| T-\frac{1}{2}, r^{\prime}\right\rangle_{N} \\
& \left\langle T, r, 2\left\|V_{0}^{1}\right\| T, r^{\prime}, 3\right\rangle_{N+1}=\frac{1}{\sqrt{2 T(T+1)}}\left\langle T-\frac{1}{2}, r\left\|V_{0}^{1}\right\| T+\frac{1}{2}, r^{\prime}\right\rangle_{N} \\
& \left\langle T, r, 2\left\|V_{0}^{1}\right\| T-1, r^{\prime}, 3\right\rangle_{N+1}=\frac{1}{\sqrt{2} T}\left\langle T-\frac{1}{2}, r\left\|V_{0}^{1}\right\| T-\frac{1}{2}, r^{\prime}\right\rangle_{N} \\
& \left\langle T, r, 4\left\|V_{0}^{1}\right\| T+1, r^{\prime}, 1\right\rangle_{N+1}=\frac{(-1)^{N}}{\sqrt{2}(T+1)}\left\langle T+\frac{1}{2}, r\left\|V_{0}^{1}\right\| T+\frac{1}{2}, r^{\prime}\right\rangle_{N} \\
& \left\langle T, r, 4\left\|V_{0}^{1}\right\| T, r^{\prime}, 1\right\rangle_{N+1}=\frac{(-1)^{N}}{\sqrt{2 T(T+1)}}\left\langle T+\frac{1}{2}, r\left\|V_{0}^{1}\right\| T-\frac{1}{2}, r^{\prime}\right\rangle_{N} \\
& \left\langle T, r, 3\left\|V_{0}^{1}\right\| T+1, r^{\prime}, 2\right\rangle_{N+1}=\frac{-1}{\sqrt{2}(T+1)}\left\langle T+\frac{1}{2}, r\left\|V_{0}^{1}\right\| T+\frac{1}{2}, r^{\prime}\right\rangle_{N} \\
& \left\langle T, r, 3\left\|V_{0}^{1}\right\| T, r^{\prime}, 2\right\rangle_{N+1}=\frac{-1}{\sqrt{2 T(T+1)}}\left\langle T+\frac{1}{2}, r\left\|V_{0}^{1}\right\| T-\frac{1}{2}, r^{\prime}\right\rangle_{N}
\end{aligned}
$$

The matrix elements between all states at intermediate steps have to be calculated. The initial values are

$$
\begin{aligned}
& \left\langle\frac{1}{2}, 1\left\|V_{0}^{1}\right\| \frac{1}{2}, 2\right\rangle=-\sqrt{3} \\
& \left\langle\frac{1}{2}, 1\left\|V_{0}^{0}\right\| \frac{1}{2}, 2\right\rangle=1 \\
& \left\langle\frac{1}{2}, 2\left\|V_{0}^{1}\right\| \frac{1}{2}, 1\right\rangle=-\sqrt{3} \\
& \left\langle\frac{1}{2}, 2\left\|V_{0}^{0}\right\| \frac{1}{2}, 1\right\rangle=-1
\end{aligned}
$$


The spectral density of the spin up Green's function can be evaluated in a similar way and is found to depend only on the matrix element of the tensor operator $V_{0}^{1}$ and not on $V_{0}^{0}$. This Green's function is the same as that of the $\alpha=1$ or $\alpha=2$ Majorana fermion Green's functions, which are equal and equivalent to the Green's function for the $\alpha=3$ Majorana fermion as a result of the $\mathrm{O}(3)$ symmetry of the model. We can deduce that $V_{0}^{1}$ is associated with the $\alpha=1,2,3$ Majorana fermions and $V_{0}^{0}$ with the $\alpha=0$ Majorana fermion. As spin down Green's function is the half the sum of the Green's functions for the Majorana fermions for $\alpha=0,3$ we can deduce that the term involving $V_{0}^{0}$ in the final formula for the spectral density of the spin down Green's function is the spectral density of the $\alpha=0$ Majorana fermion (apart from a factor $1 / 2$ ) and, similarly, the term in $V_{0}^{1}$ is the spectral density of the $\alpha=1,2,3$ Majorana fermions.

TABLE I. Many-body excitation energies and their corresponding degeneracies $(d g)$ of the two channel Kondo model corresponding to the single particle spectrum at the low-energy fixed point. The energy levels without primes correspond to sector $\mathrm{A}$, and those with primes to sector B.

\begin{tabular}{cccc}
\hline \hline$E_{\text {ex }} /\left(\pi v_{\mathrm{F}} / l\right)$ & $\sum_{k} n_{k} \epsilon_{k}$ & $d g$ & total $d g$ \\
\hline 0 & 0 & 2 & 2 \\
\hline $1 / 8$ & $0^{\prime}$ & 4 & 4 \\
\hline $1 / 2$ & $\varepsilon_{1 / 2}$ & 10 & 10 \\
\hline $5 / 8$ & $\varepsilon_{1 / 2}^{\prime}$ & 12 & 12 \\
\hline 1 & $\varepsilon_{1}$ & 6 & 26 \\
\hline $9 / 8$ & $2 \varepsilon_{1 / 2}$ & 20 & 32 \\
\hline $3 / 2$ & $\varepsilon_{1}^{\prime}$ & 20 & \\
& $2 \varepsilon_{1 / 2}^{\prime}$ & 12 & \\
& $\varepsilon_{3 / 2}$ & 10 & \\
& $\varepsilon_{1}+\varepsilon_{1 / 2}$ & 30 & \\
& $3 \varepsilon_{1 / 2}$ & 20 & \\
\hline $13 / 8$ & $\varepsilon_{3 / 2}^{\prime}$ & 12 & \\
& $\varepsilon_{1}^{\prime}+\varepsilon_{1 / 2}^{\prime}$ & 60 & 76 \\
\hline \hline
\end{tabular}


${ }^{1}$ P. Coleman and A. J. Schofield, Phys. Rev. Lett. 75 (1995) 2184.

${ }^{2}$ G.-M. Zhang and A. C. Hewson, Phys. Rev. Lett. 76, (1996 2137); Phys. Rev. B 54 (1996) 1169.

${ }^{3}$ R. Bulla and A.C. Hewson, Z. Phys. B 104 (1997) 333.

${ }^{4}$ R. Bulla, A.C. Hewson and G-M Zhang, Phys. Rev. B 56 (1997) 11721.

${ }^{5}$ P. Coleman, L. Ioffe, and A. M. Tsvelik, Phys. Rev. B 52 (1995) 6611.

${ }^{6}$ D. L Cox and A. Zawadowski, Advances in Physics 47 (1998) 599.

${ }^{7}$ A. J. Schofield, Phys. Rev. B 55 (1997) 5627.

${ }^{8}$ A. C. Hewson, Phys. Rev. Lett. 70 (1993) 4007; Advances in Physics 43, (1994) 543.

${ }^{9}$ A. C. Hewson, The Kondo Problem to Heavy Fermions. Cambridge University Press (1993).

${ }^{10}$ I. Affleck and A. W. W. Ludwig, Nucl. Phys. B 360 (1991) 641: Phys. Rev. B 48 (1993) 7297; I. Affleck, A. W. W. Ludwig, H. -B. Pang, D. L. Cox, Phys. Rev. B, 45 (1992) 7918.

${ }^{11}$ V. G. Knizhnik and A. B. Zamolodchikov, Nucl. Phys. B 247 (1984) 83.

${ }^{12}$ P. Ginsparg in Fields, Strings and Critical Phenomena, Les Houches XLIX, E. Brèzin and J. Zinn-Justin (Eds), (North Holland, Amsterdam 1990).

${ }^{13}$ P. Nozières and A. Blandin, J. de Physique 41 (1980) 193.

14 J. M. Maldacena and A. W. W. Ludwig, Nucl. Phys. B, 506 (1997) 565.

15 J. Ye, Nuclear Physics B 512 (1998) 543.

16 A. Georges and A. Sengupta, Nuclear Physics B (Proc. Suppl.) 58105.

${ }^{17}$ H. B. Pang, D. L. Cox, Phys. Rev. B, 44 (1991) 9454.

18 K.G. Wilson, Rev. Mod. Phys. 47 (1975) 773.

${ }^{19}$ H.R. Krishna-murthy, J.W. Wilkins and K.G. Wilson, Phys. Rev. B(1980) 21, 1003 \& 1044.

${ }^{20}$ O. Sakai, Y. Shimizu and T. Kasuya, J. Phys. Soc. Japan, 58 (1989) 3666.

${ }^{21}$ T.A. Costi, A.C. Hewson and V. Zlatic, J. Phys.: Cond. Mat. 6 (1994) 2519.

${ }^{22}$ S. Bradley, R. Bulla and A.C. Hewson, Physica B 259-261, (1999) 362.

${ }^{23}$ O. Sakai, Y. Shimizu and N. Kaneko, Physica B 186-188 (1993) 323.

${ }^{24}$ C.M. Varma, P.B. Littlewood, S. Schmitt-Rink, E. Abrahams, and A.E. Ruckenstein, Phys. Rev. Lett. 63 (1989) 1996. 


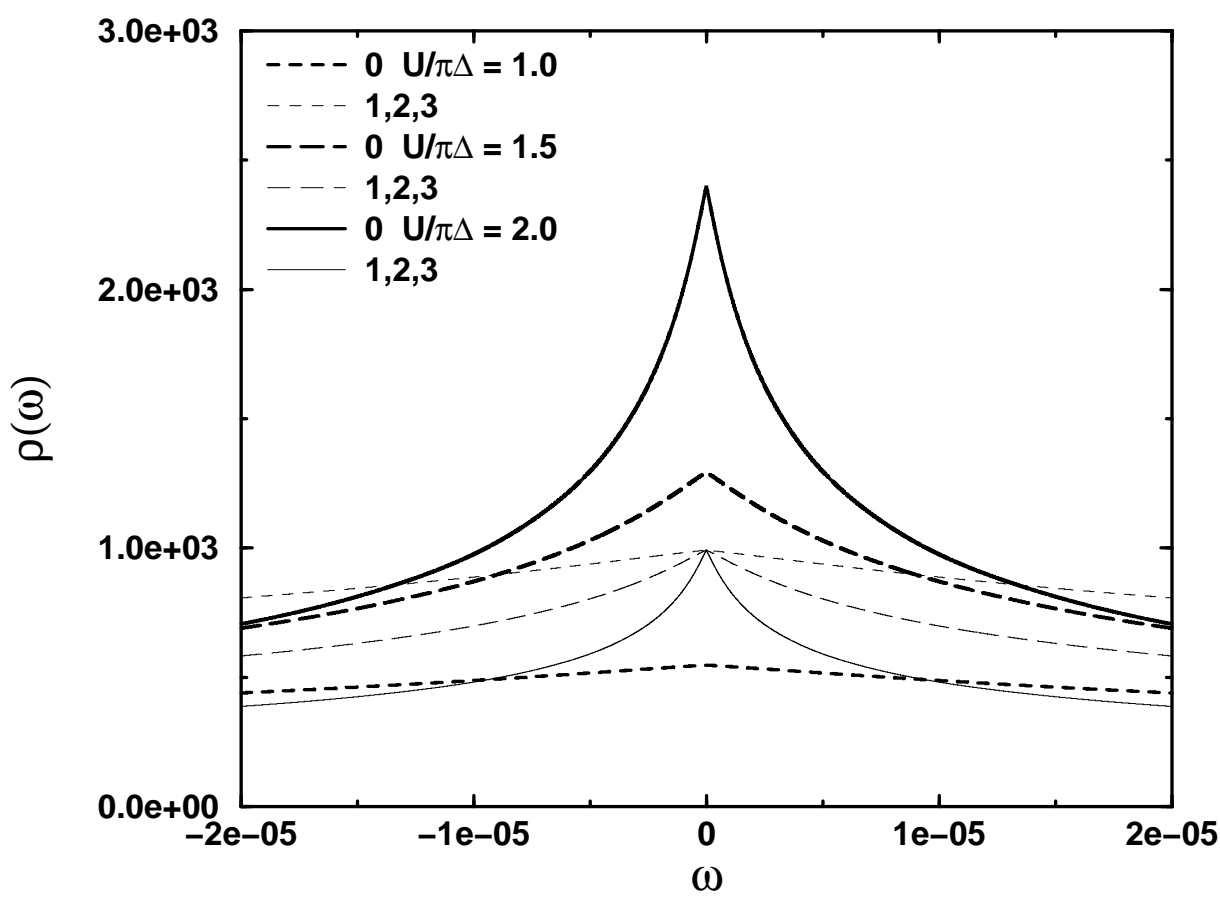

FIG. 1. The spectral density $\rho(\omega)$ of the Majorana fermion impurity Green's function in the marginal Fermi liquid situation: $V=0.01414, V_{0}=0.0\left(\Delta=10^{-4} \pi, \Delta_{0}=0.0\right)$.

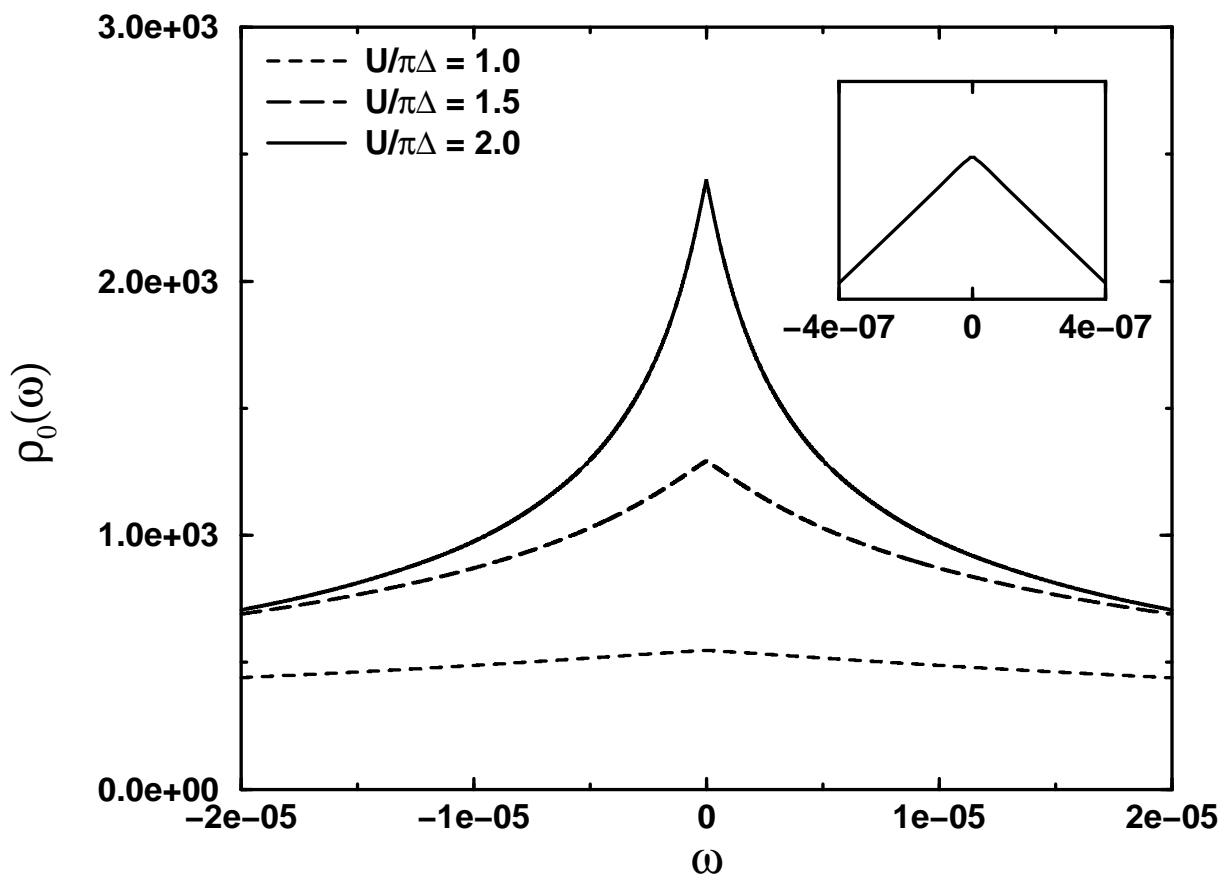

FIG. 2. The spectral density $\rho_{0}(\omega)$ of the $\alpha=0$ Majorana fermion Green's function in the marginal Fermi liquid situation: $V=0.01414, V_{0}=0.0\left(\Delta=10^{-4} \pi, \Delta_{0}=0.0\right)$. The inset shows the $|\omega|$ dependence near the Fermi level. 


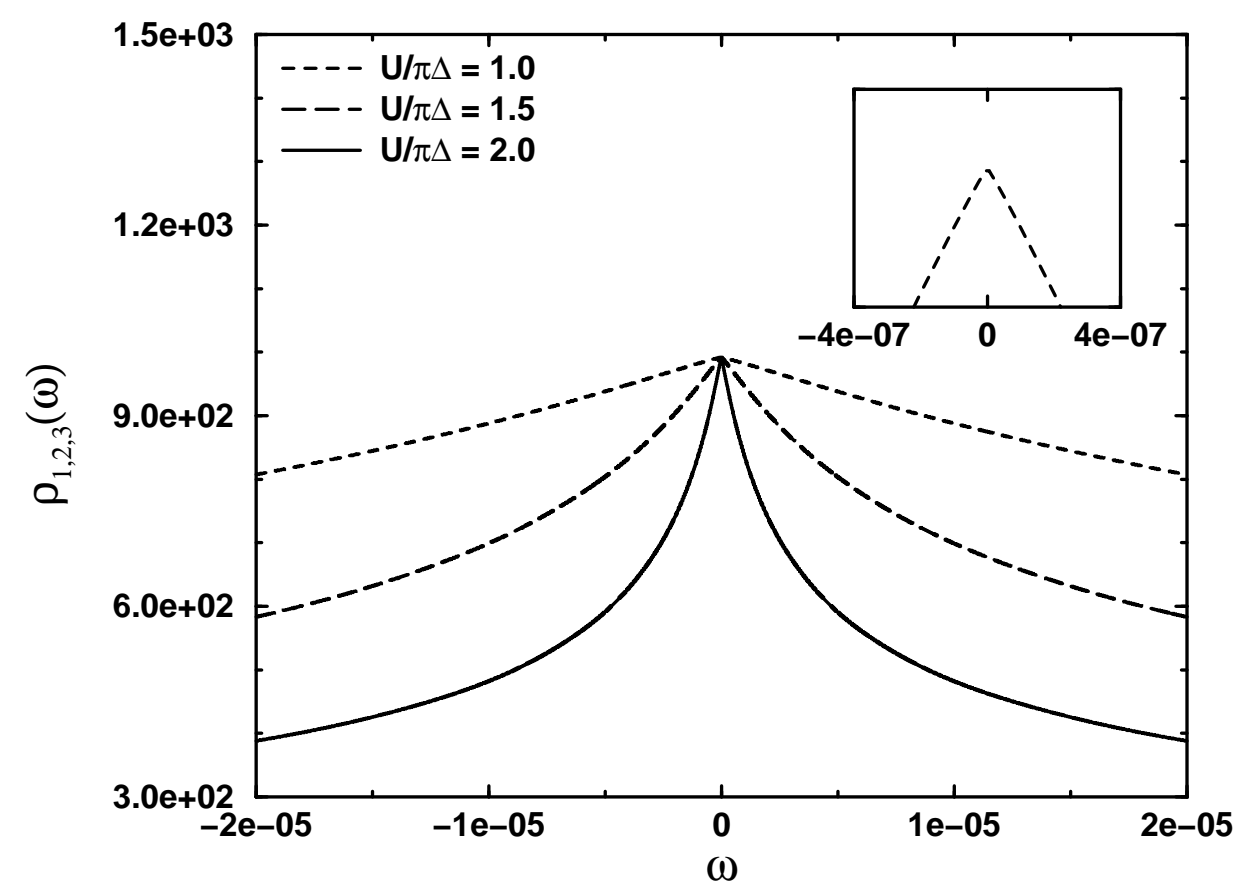

FIG. 3. The spectral density $\rho_{1,2,3}(\omega)$ of the $\alpha=1,2,3$ Majorana fermion Green's function in the marginal Fermi liquid situation: $V=0.01414, V_{0}=0.0\left(\Delta=10^{-4} \pi, \Delta_{0}=0.0\right)$. The inset shows the $|\omega|$ dependence near the Fermi level. $\rho_{1,2,3}(0)$ is within $2 \%$ of the value given by the Friedel sum rule.

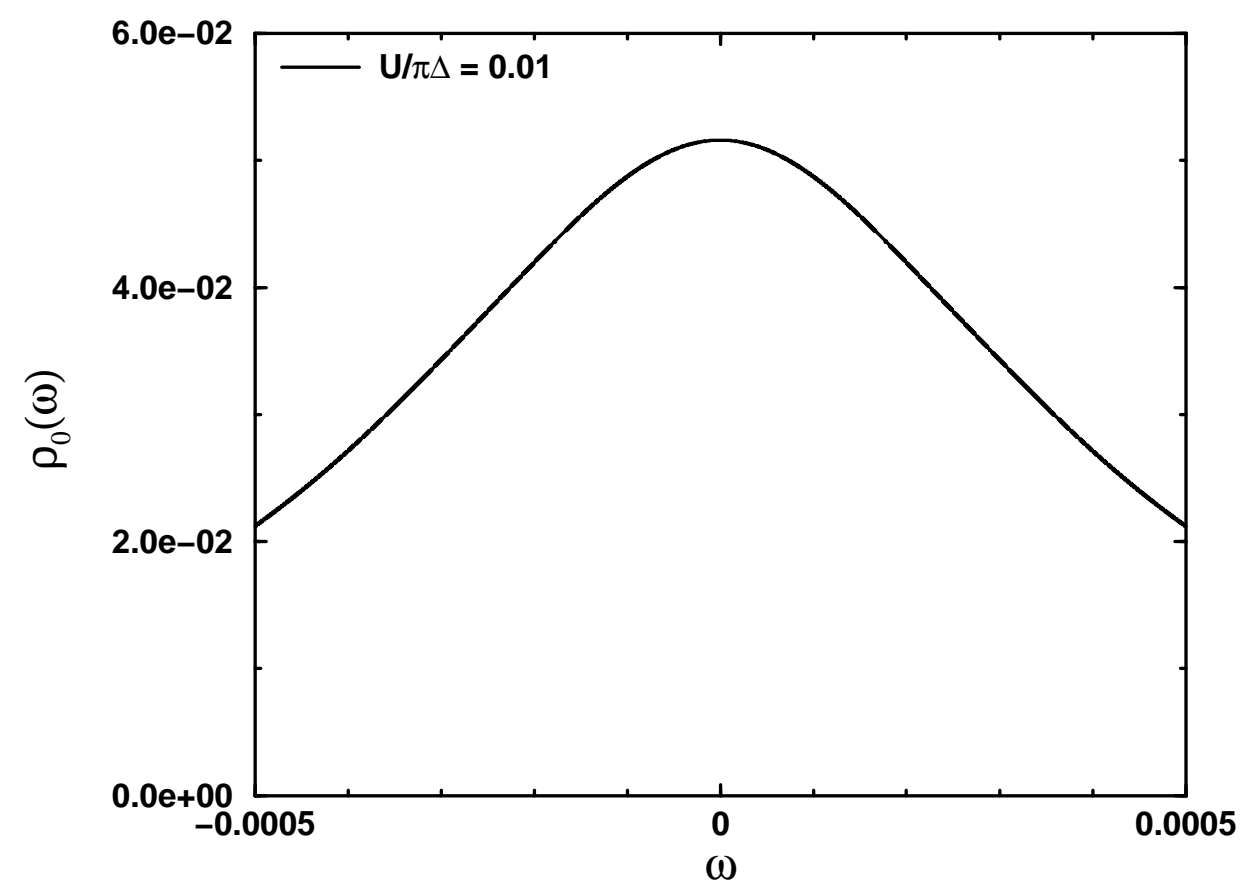

FIG. 4. The spectral density $\rho_{0}(\omega)$ of the $\alpha=0$ Majorana fermion Green's function in the marginal Fermi liquid situation: $V=0.01414, V_{0}=0.0\left(\Delta=10^{-4} \pi, \Delta_{0}=0.0\right)$, indicating the influence of $w^{2}$ corrections for small $U / \pi \Delta$. 


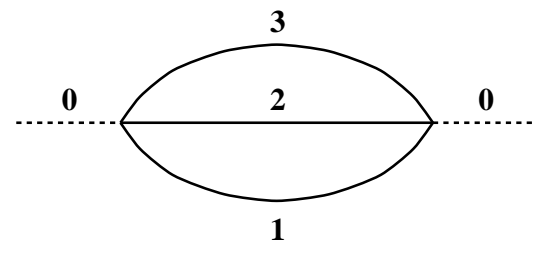

(a)

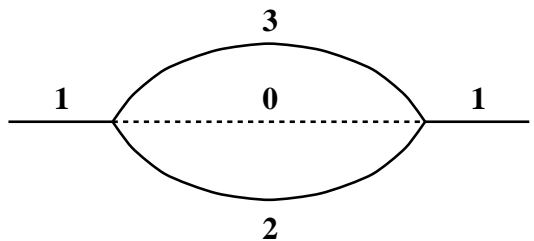

(b)

FIG. 5.

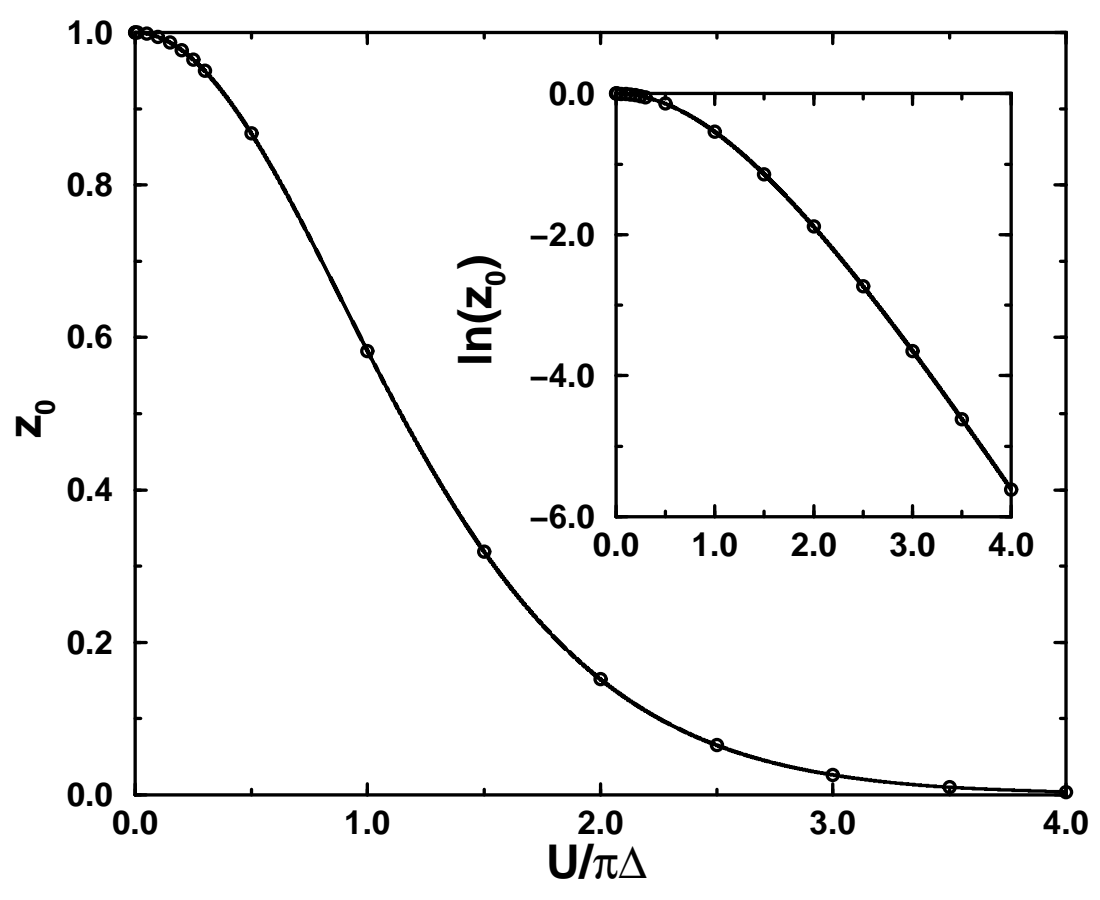

FIG. 6. The wavefunction renormalization factor, $z_{0}$, determined by the coefficient of $\delta(\omega)$ in the spectral density, $\rho_{0}(\omega)$. Exponential behaviour for large $U / \pi \Delta$ is indicated by the linear region of the inset $\ln \left(z_{0}\right)$ plot.

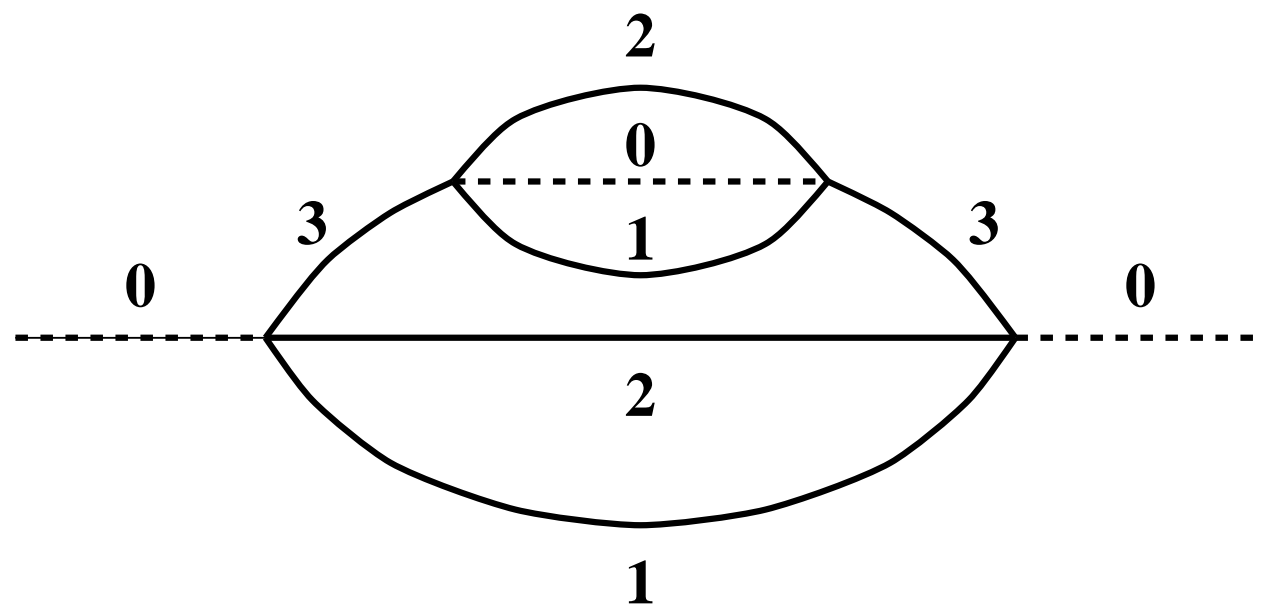

FIG. 7. 


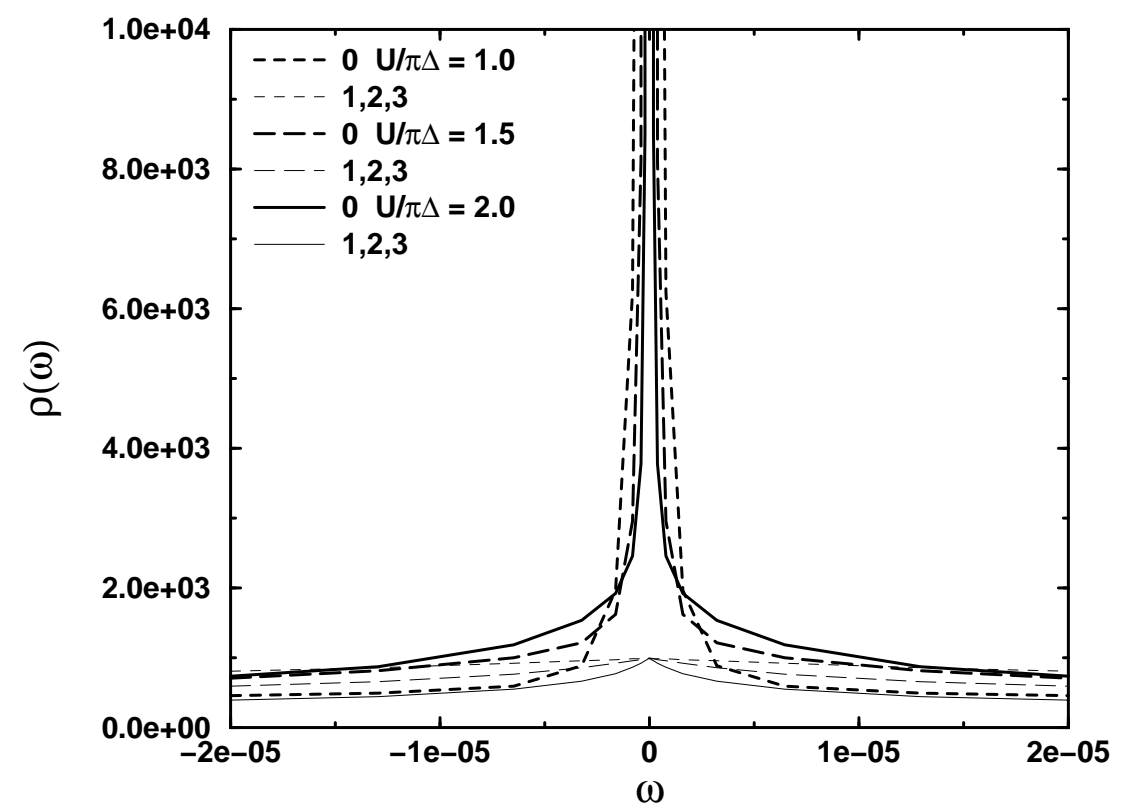

FIG. 8. The spectral density $\rho(\omega)$ of the Majorana fermion impurity Green's function in the Fermi liquid situation: $V=0.01414, V_{0}=0.00014\left(\Delta=10^{-4} \pi, \Delta_{0}=10^{-8} \pi\right)$.

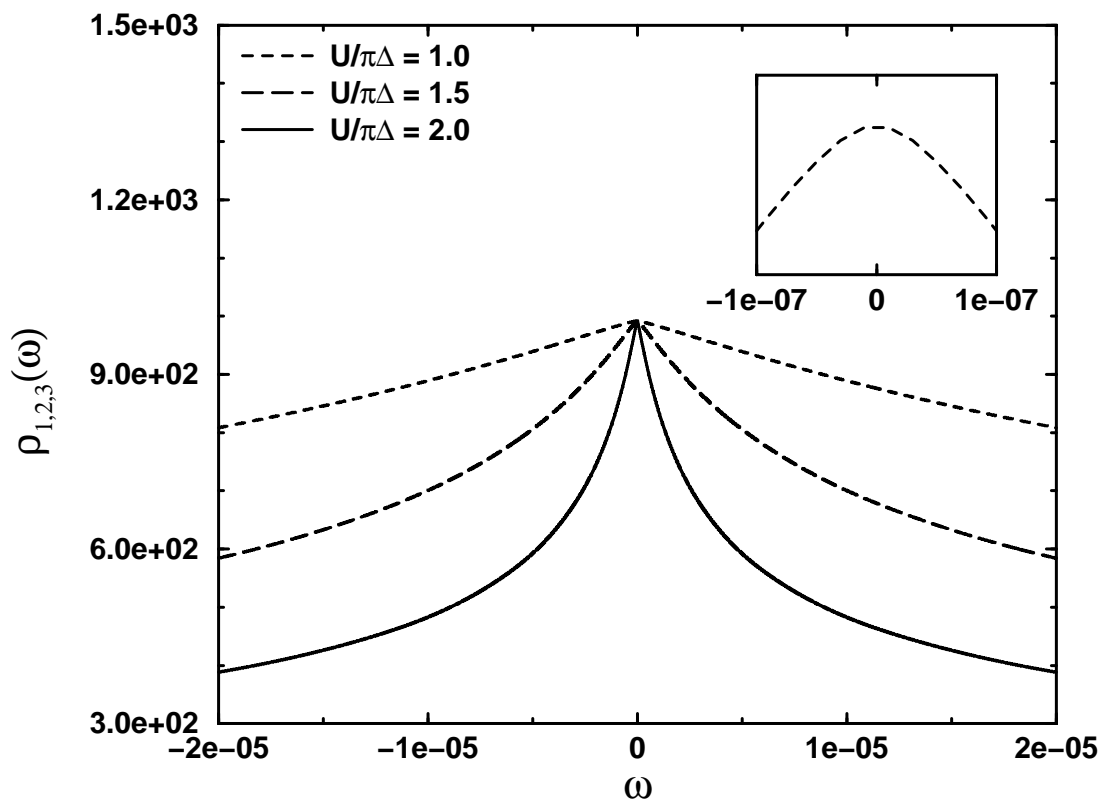

FIG. 9. The spectral density $\rho_{1,2,3}(\omega)$ of the $\alpha=1,2,3$ Majorana fermion Green's function in the Fermi liquid situation: $V=0.01414, V_{0}=0.00014\left(\Delta=10^{-4} \pi, \Delta_{0}=10^{-8} \pi\right)$. The inset shows the region of the spectrum near the Fermi level where flattening consistent with $\omega^{2}$ behaviour is observed. $\rho_{1,2,3}(0)$ is within $2 \%$ of the value given by the Friedel sum rule. 


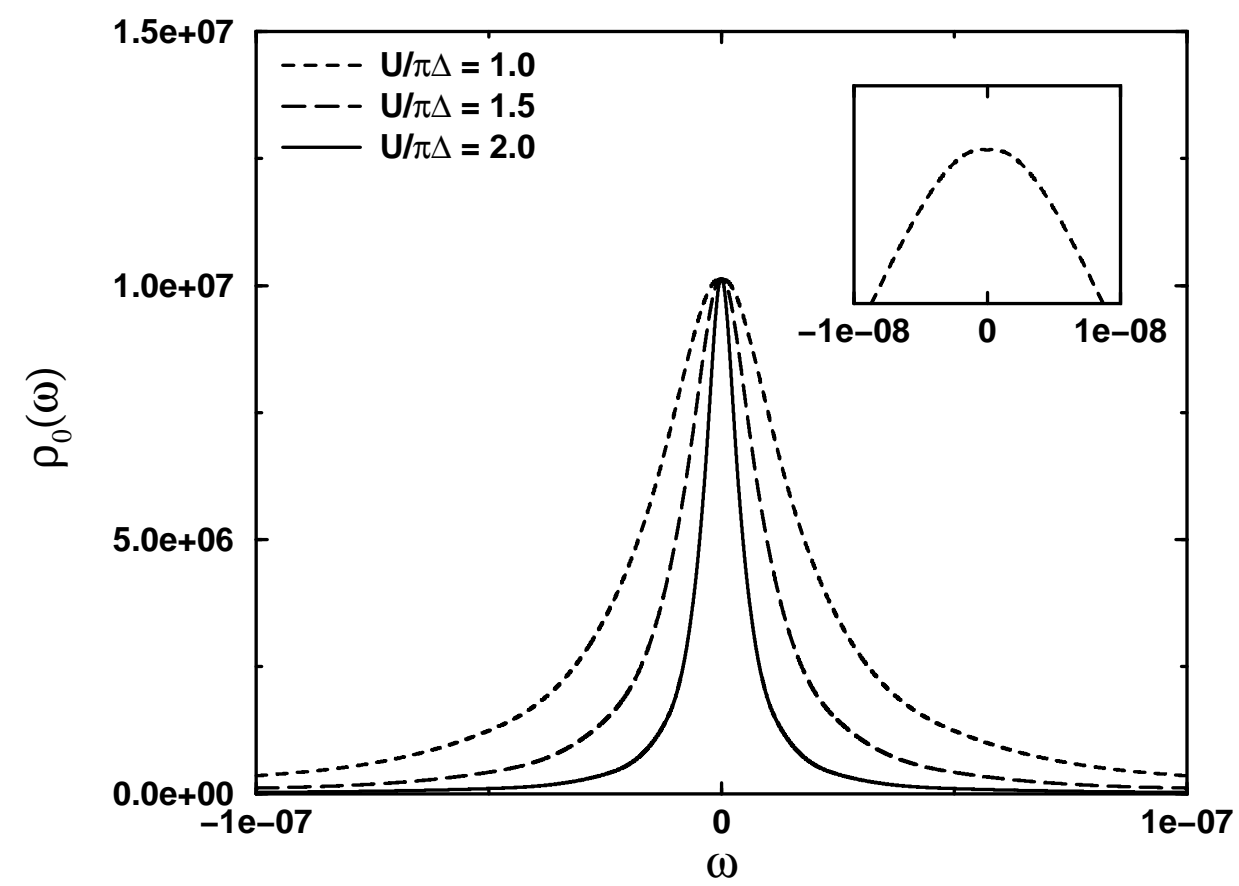

FIG. 10. The spectral density $\rho_{0}(\omega)$ of the $\alpha=0$ Majorana fermion Green's function in the Fermi liquid situation: $V=0.01414, V_{0}=0.00014\left(\Delta=10^{-4} \pi, \Delta_{0}=10^{-8} \pi\right)$. The inset shows the $\omega^{2}$ dependence near the Fermi level. $\rho_{0}(0)$ is within $2 \%$ of the value given by the Friedel sum rule.

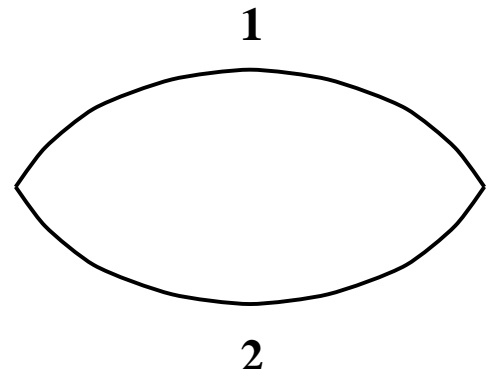

(a)

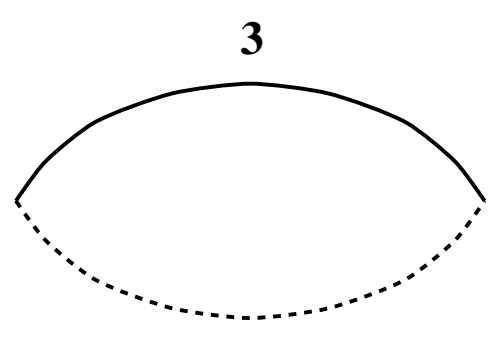

$\mathbf{0}$

(b)

FIG. 11. 


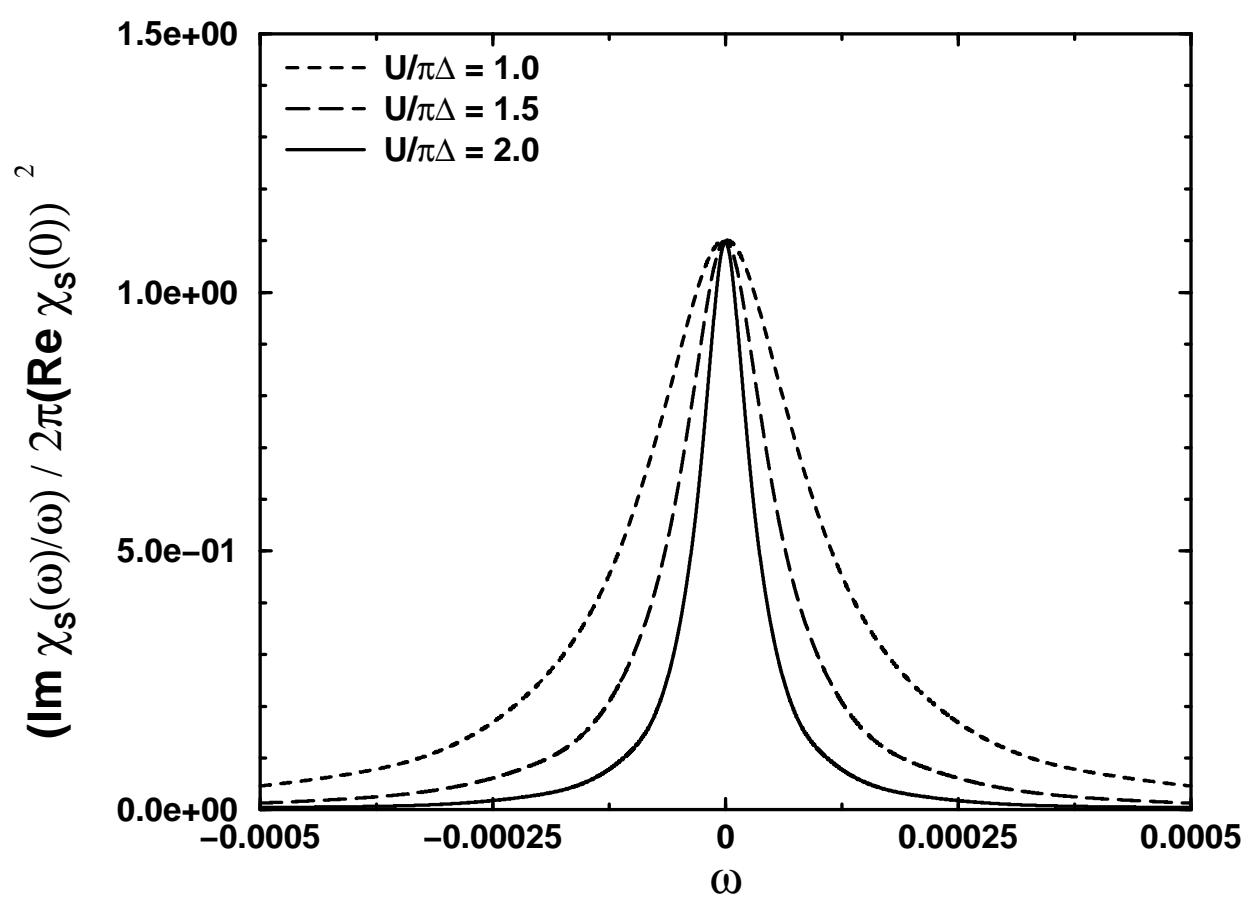

FIG. 12. $\left(\operatorname{Im} \chi_{s}(\omega) / \omega\right) / 2 \pi\left(\operatorname{Re} \chi_{s}(0)\right)^{2}$ for the standard model: $V=V_{0}=0.01414\left(\Delta=\Delta_{0}=10^{-4} \pi\right)$.

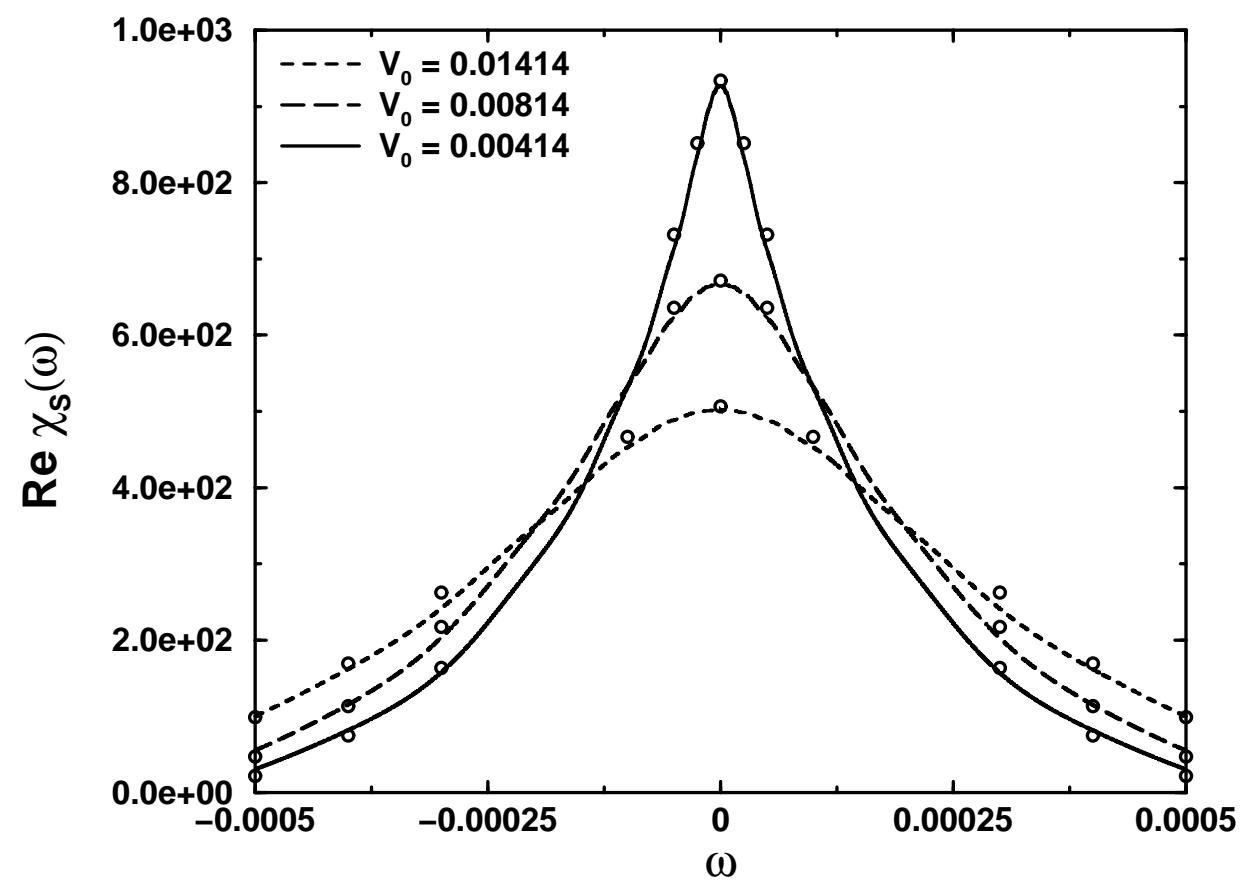

FIG. 13. The real part of the dynamic spin susceptibility $\chi_{s}(\omega)$ for the non-interacting model: $V=0.01414\left(\Delta=10^{-4} \pi\right)$, $U / \pi \Delta=0.0$. The circles denote analytic values. 


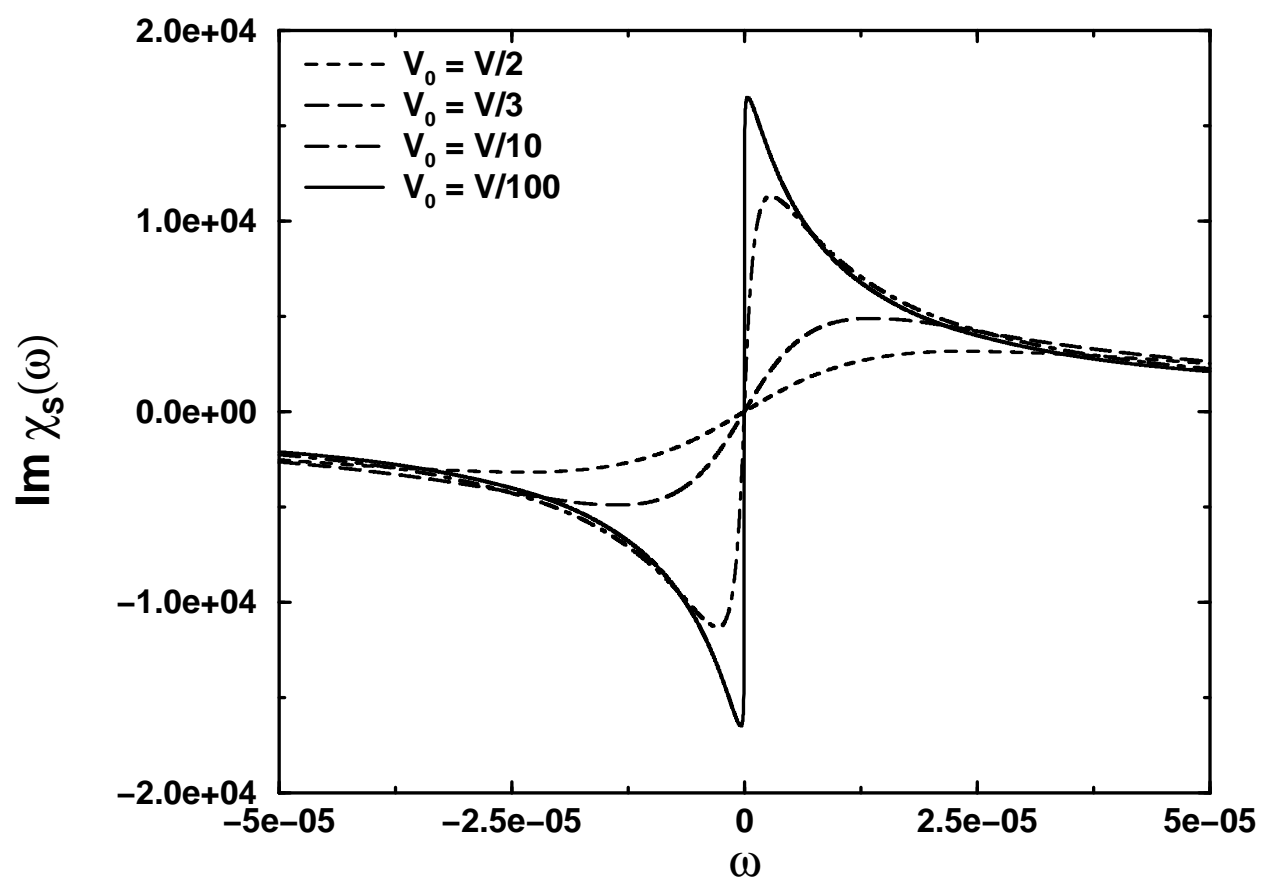

FIG. 14. The imaginary part of the dynamic spin susceptibility $\chi_{s}(\omega)$ in the Fermi liquid situation: $V=0.01414\left(\Delta=10^{-4} \pi\right), U / \pi \Delta=1.5$.

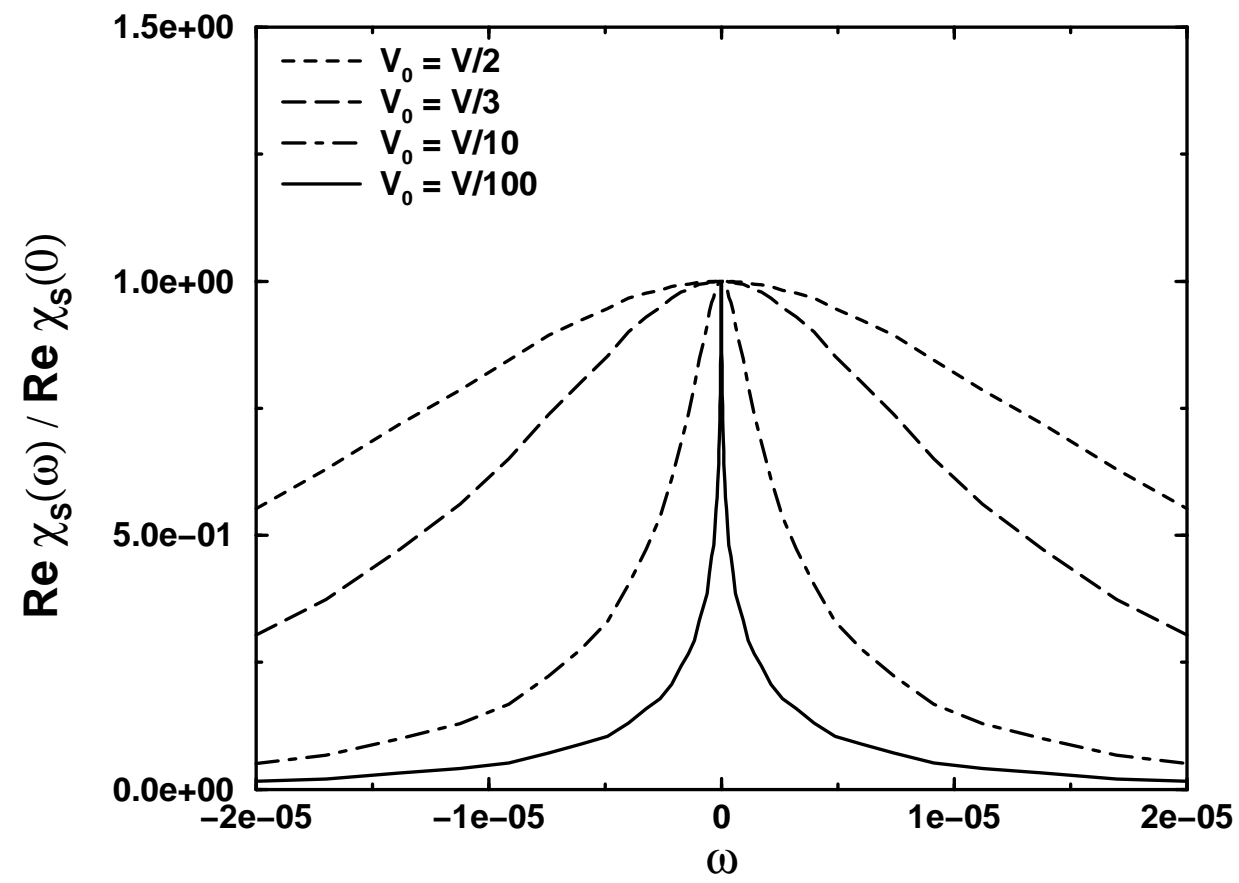

FIG. 15. The real part of the dynamic spin susceptibility $\chi_{s}(\omega) / \chi_{s}(0)$ in the Fermi liquid situation: $V=0.01414\left(\Delta=10^{-4} \pi\right), U / \pi \Delta=1.5$. 


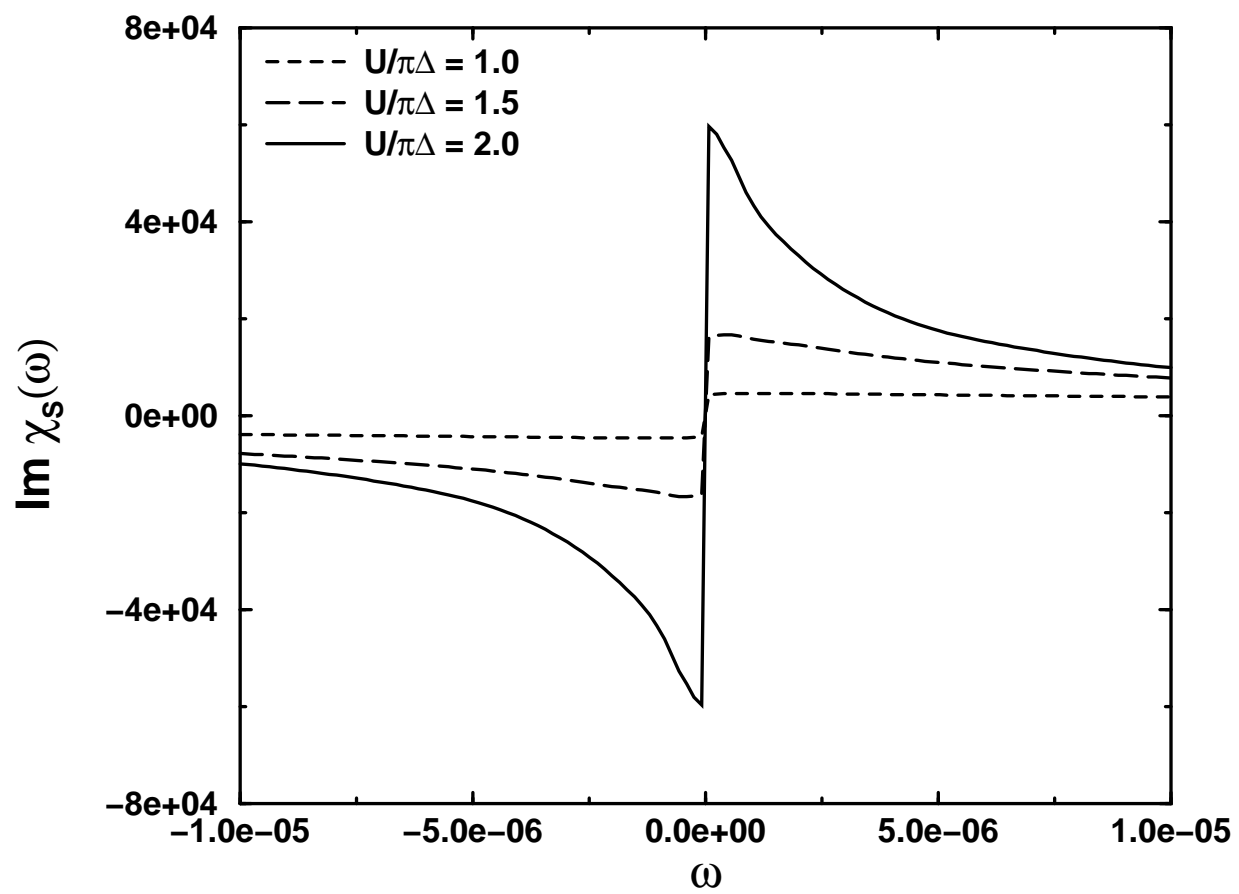

FIG. 16. The imaginary part of the dynamic spin susceptibility $\chi_{s}(\omega)$ in the marginal Fermi liquid situation: $V=0.01414, V_{0}=0.0\left(\Delta=10^{-4} \pi, \Delta_{0}=0.0\right)$.

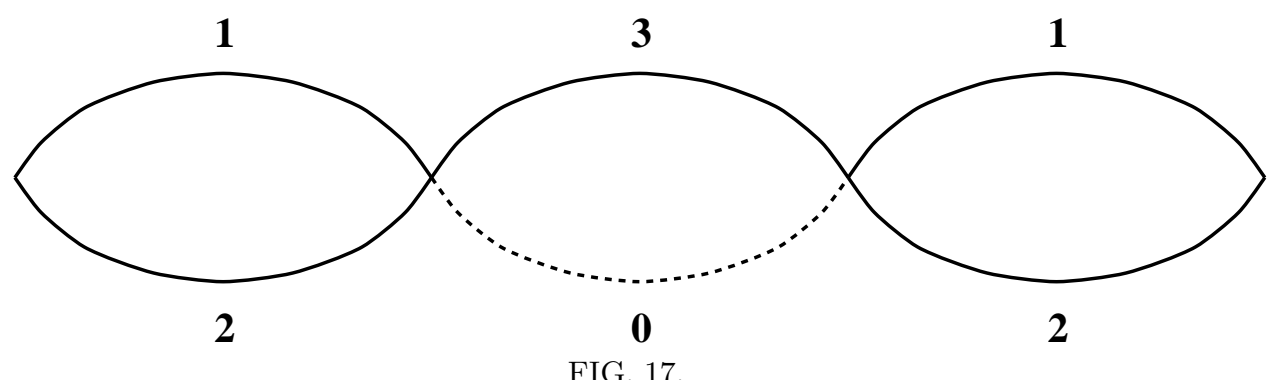

FIG. 17. 


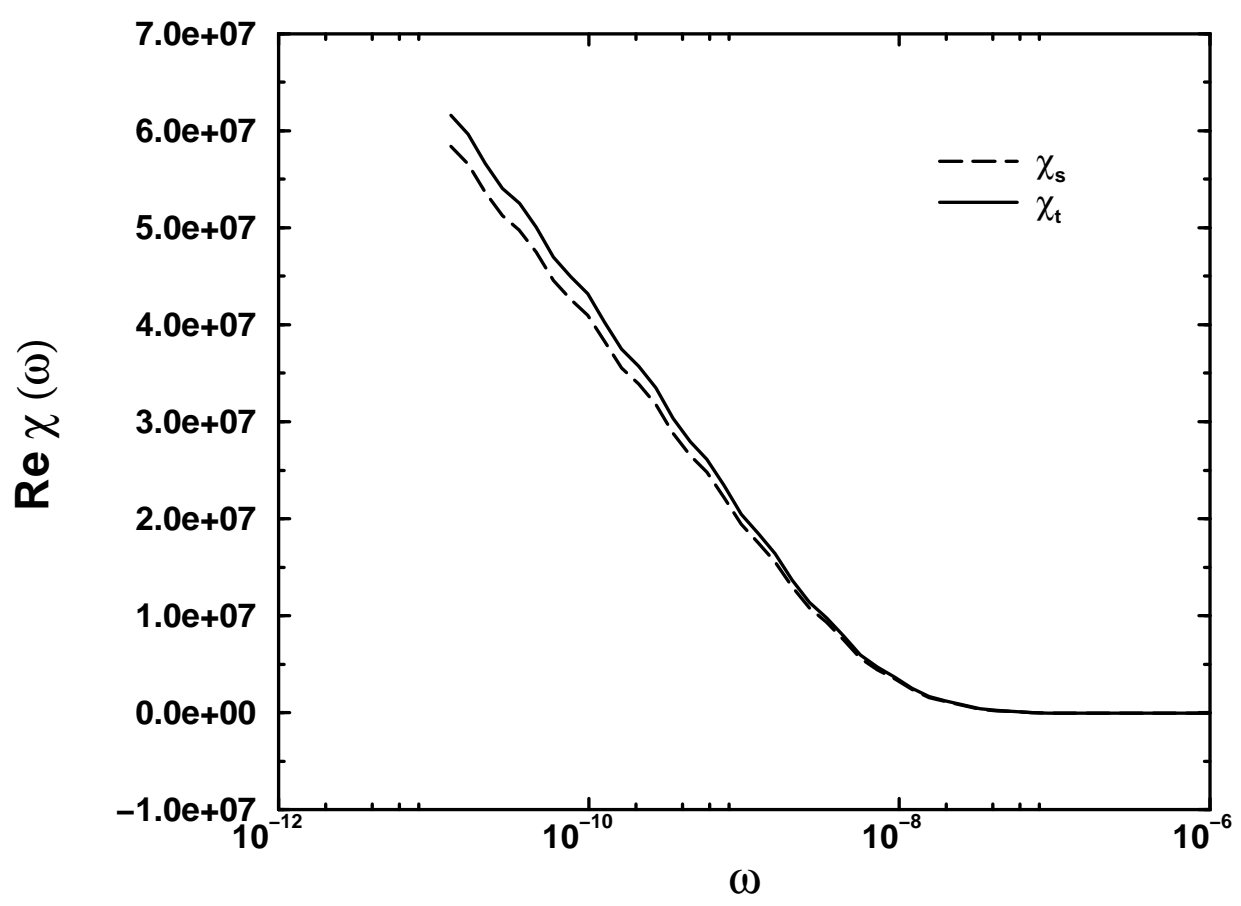

FIG. 18. The real part of the dynamic spin susceptibilities $\chi_{\mathbf{t}}(\omega)$ and $\chi_{s}(\omega)$ in the marginal Fermi liquid situation: $V=0.01414, V_{0}=0.0\left(\Delta=10^{-4} \pi, \Delta_{0}=0.0\right), U / \pi \Delta=4.0$, displaying linear dependence on $\ln (\omega)$ in the low energy region. 